\title{
MINIMAL SUPERSOLUTIONS OF CONVEX BSDES UNDER CONSTRAINTS *
}

\author{
Gregor Heyne $^{1}$, Michael Kupper ${ }^{2}$, Christoph Mainberger ${ }^{1}$ \\ AND LUDOVIC TANGPI ${ }^{3}$
}

\begin{abstract}
We study supersolutions of a backward stochastic differential equation, the control processes of which are constrained to be continuous semimartingales of the form $\mathrm{d} Z=\Delta \mathrm{d} t+\Gamma \mathrm{d} W$. The generator may depend on the decomposition $(\Delta, \Gamma)$ and is assumed to be positive, jointly convex and lower semicontinuous, and to satisfy a superquadratic growth condition in $\Delta$ and $\Gamma$. We prove the existence of a supersolution that is minimal at time zero and derive stability properties of the non-linear operator that maps terminal conditions to the time zero value of this minimal supersolution such as monotone convergence, Fatou's lemma and $L^{1}$-lower semicontinuity. Furthermore, we provide duality results within the present framework and thereby give conditions for the existence of solutions under constraints.
\end{abstract}

Mathematics Subject Classification. 60H20, 60H30.

Received October 6, 2014. Revised March 2nd, 2016. Accepted April 18, 2016.

\section{INTRODUCTION}

On a filtered probability space, the filtration of which is generated by a $d$-dimensional Brownian motion, we are interested in quadruplets $(Y, Z, \Delta, \Gamma)$ of processes such that, for all $0 \leq s \leq t \leq T$, the system

$$
\begin{aligned}
& Y_{s}-\int_{s}^{t} g_{u}\left(Y_{u}, Z_{u}, \Delta_{u}, \Gamma_{u}\right) \mathrm{d} u+\int_{s}^{t} Z_{u} \mathrm{~d} W_{u} \geq Y_{t}, \quad Y_{T} \geq \xi, \\
& Z_{t}=z+\int_{0}^{t} \Delta_{u} \mathrm{~d} u+\int_{0}^{t} \Gamma_{u} \mathrm{~d} W_{u}
\end{aligned}
$$

is satisfied. Here, for $\xi$ a terminal condition, $Y$ is the càdlàg value process and $Z$ the continuous control process with decomposition $(\Delta, \Gamma)$. The generator $g$ is assumed to be jointly convex and may depend on the decomposition of the continuous semimartingale $Z$. It is our objective to give conditions ensuring that the set $\mathcal{A}(\xi, g, z)$, consisting of all admissible pairs $(Y, Z)$ satisfying (1.1), henceforth called supersolution of the

Keywords and phrases. Supersolutions of backward stochastic differential equations, gamma constraints, minimality under constraints, duality.

* Financial support: MATHEON project E.2.

1 Humboldt-Universität zu Berlin, Unter den Linden 6, 10099 Berlin, Germany. heyne@math.hu-berlin.de; mainberg@math.hu-berlin.de

2 University of Konstanz, Universitätsstr. 10, 78457 Konstanz, Germany. kupper@uni-konstanz.de

3 University of Vienna, Faculty of Mathematics, Oskar-Morgenstern-Platz 1, 1090 Vienna. ludovic.tangpi@univie.ac.at 
backward stochastic differential equation (BSDE) under gamma and delta constraints, contains elements $(\hat{Y}, \hat{Z})$ that are minimal at time zero. Furthermore, we give conditions relying on BSDE duality for the existence of solutions under constraints.

Finding the minimal initial value of a supersolution under constraints is closely related to the superreplication problem in a financial market under gamma constraints, first studied in Soner and Touzi [21]. Indeed, the classical gamma constraints can be incorporated into our more general framework by setting the generator to $+\infty$ whenever the diffusion part $\Gamma$ is outside a predetermined interval. In Soner and Touzi [21], the decomposition parts of the trading strategies are assumed to be bounded and under similar assumptions, Cheridito et al. [3] focus on the multidimensional case. In both papers the problem is formulated in a dynamic setting, allowing the authors to use dynamic programming tools to identify the value functions as unique viscosity solutions of parabolic partial differential equations. The present paper in contrast focuses on the static case and studies the problem using purely probabilistic tools. Moreover, instead of a priori bounding the components of the control process, we opted for incorporating a growth condition on the generator which in turn ensures that our controls belong to suitable spaces. Let us briefly outline the idea behind our approach. In a nutshell, inspired by the methods first used in Drapeau et al. [9] and then later in Heyne et al. [13], we begin by considering the operator $\mathcal{E}_{0}^{g}(\xi, z):=\inf \left\{Y_{0}:(Y, Z) \in \mathcal{A}(\xi, g, z)\right\}$ where $z \in \mathbb{R}^{1 \times d}$ is the initial value of controls. We then show that the set of supersolutions $(\hat{Y}, \hat{Z})$ satisfying $\hat{Y}_{0}=\mathcal{E}_{0}^{g}(\xi, z)$ is non-empty. In order to do so, we impose a superquadratic growth condition in the decomposition parts $(\Delta, \Gamma)$ of controls on the generator $g$, reflecting a penalization of rapid changes in control values and accounting for the expression "Delta- and Gamma-Constraints". The consequence is twofold. First, it ensures that the sequence of stochastic integrals $\left(\int Z^{n} \mathrm{~d} W\right)$ corresponding to the minimizing sequence $Y_{0}^{n} \downarrow \mathcal{E}_{0}^{g}(\xi, z)$ is bounded in $\mathcal{H}^{2}$. Drawing from compactness results for the space of martingales $\mathcal{H}^{2}$ given in Delbaen and Schachermayer [8], we obtain our candidate control process $\hat{Z}$ as the limit of a sequence in the asymptotic convex hull of $\left(Z^{n}\right)$. At this point it is crucial to preserve the continuous semimartingale structure of the limit object, that is $\hat{Z}=z+\int \hat{\Delta} \mathrm{d} u+\int \hat{\Gamma} \mathrm{d} W$. Here the first novelty of this paper comes into play since, although following the ideas used in Drapeau et al. [9], it is a priori not clear that the candidate control has the right structure. To achieve this, we prove two auxiliary results by using once more the aforementioned growth condition on $g$.

In a next step, we provide stability results of $\xi \mapsto \mathcal{E}_{0}^{g}(\xi, z)$, the non-linear operator that maps a terminal condition to the value of the minimal supersolution at time zero, such as monotone convergence, Fatou's lemma or $L^{1}$-lower semicontinuity. This, together with convexity, gives way to a dual representation of $\mathcal{E}_{0}^{g}$ as a consequence of the Fenchel-Moreau theorem, which is the second main novel contribution of this work. Indeed, we use purely probabilistic methods in order to characterize the conjugate $\mathcal{E}_{0}^{*}$ in terms of the decomposition parts of the controls and show that $\mathcal{E}_{0}^{*}$ is always attained. Note that, in contrast to Drapeau et al. [10], in the presence of constraints identifying the convex conjugate $\mathcal{E}_{0}^{*}$ is technically more involved. In particular, for the case of a quadratic generator we show that it is possible to explicitly compute the conjugate by means of classical calculus of variations methods, giving additional structural insight into the problem. If we assume in turn the existence of an optimal subgradient such that $\mathcal{E}_{0}^{g}(\xi, z)$ is attained in its dual representation, we can prove that the associated BSDE with parameters $(\xi, g)$ admits a solution under constraints. Our duality results extend those of Delbaen et al. [6] and Drapeau et al. [10] obtained in the unconstrained case as the existence of constraints require new methods in order to characterize the convex conjugate.

Before we continue, let us briefly discuss the existing literature on the subject. Ever since the seminal paper Pardoux and Peng [18], an extensive amount of work has been done in the field of BSDEs, resulting in such important contributions as for instance El Karoui et al. [11], Kobylanski [17], Briand and Hu [1]. We refer the reader to Peng [19], Drapeau et al. [9] for a more thorough treatment of the literature concerning solutions and in particular supersolutions of BSDEs. There are many works dealing with optimization or (super-)replication under constraints, see for instance Cvitanic and Karatzas [5], Jouini and Kallal [14], Broadie et al. [2] and references therein, but the notion of gamma constraints in the context of superhedging was introduced in Soner and Touzi [21] and then studied in a multi-dimensional setting in Cheridito et al. [3]. We would also like to refer the reader to Cheridito et al. [4], where the authors treat the related system of BSDEs and SDEs in a 
more abstract fashion, whereas the more recent work Soner et al. [22] provides a dual characterization of the superreplication problem.

The remainder of this paper is organized as follows. Setting and notations are specified in Section 2. A precise definition of supersolutions under gamma and delta constraints is then given in Section 3, along with existence and stability results. We conclude this work with duality results in Section 4.

\section{Setting And notations}

We consider a filtered probability space $\left(\Omega, \mathcal{F},\left(\mathcal{F}_{t}\right)_{t \geq 0}, P\right)$, where the filtration $\left(\mathcal{F}_{t}\right)$ is generated by a $d$ dimensional Brownian motion $W$ and is assumed to satisfy the usual conditions. For some fixed time horizon $T>0$ the set of $\mathcal{F}_{T}$-measurable random variables is denoted by $L^{0}$, where random variables are identified in the $P$-almost sure sense. By $L^{p}$ we furthermore denote the set of random variables in $L^{0}$ with finite $p$-norm, for $p \in[1,+\infty]$. Inequalities and strict inequalities between any two random variables or processes $X^{1}$ and $X^{2}$ are understood in the $P$-almost sure or in the $P \otimes \mathrm{d} t$-almost everywhere sense, respectively. We denote by $\mathcal{T}$ the set of stopping times with values in $[0, T]$ and hereby call an increasing sequence of stopping times $\left(\tau^{n}\right)$ such that $P\left[\bigcup_{n}\left\{\tau^{n}=T\right\}\right]=1$ a localizing sequence of stopping times. For $m, n \in \mathbb{N}$ we denote by $|\cdot|$ the Euclidean norm on $\mathbb{R}^{m \times n}$, that is $|x|=\left(\sum_{i, j} x_{i j}^{2}\right)^{\frac{1}{2}}$. By $\mathcal{S}:=\mathcal{S}(\mathbb{R})$ we denote the set of càdlàg progressively measurable processes $Y$ with values in $\mathbb{R}$. For $p \in\left[1,+\infty\right.$ [, we further denote by $\mathcal{H}^{p}$ the set of càdlàg local martingales $M$ with finite $\mathcal{H}^{p}$-norm on $[0, T]$, that is $\|M\|_{\mathcal{H}^{p}}:=E\left[\langle M, M\rangle_{T}^{p / 2}\right]^{1 / p}<\infty$. By $\mathcal{L}^{p}:=\mathcal{L}^{p}(W)$ we denote the set of $\mathbb{R}^{1 \times d_{-}}$ valued, progressively measurable processes $Z$ such that $\int Z \mathrm{~d} W \in \mathcal{H}^{p}$, that is, $\|Z\|_{\mathcal{L}^{p}}:=E\left[\left(\int_{0}^{T}\left|Z_{s}\right|^{2} \mathrm{~d} s\right)^{p / 2}\right]^{1 / p}$ is finite. For $Z \in \mathcal{L}^{p}$, the stochastic integral $\int Z \mathrm{~d} W$ is well defined, see [20], and is by means of the BurkholderDavis-Gundy inequality ([20], Thm. 48) a continuous martingale. We further denote by $\mathcal{L}:=\mathcal{L}(W)$ the set of $\mathbb{R}^{1 \times d}$-valued, progressively measurable processes $Z$ such that there exists a localizing sequence of stopping times $\left(\tau^{n}\right)$ with $Z 1_{\left[0, \tau^{n}\right]} \in \mathcal{L}^{1}$, for all $n \in \mathbb{N}$. For $Z \in \mathcal{L}$, the stochastic integral $\int Z \mathrm{~d} W$ is well defined and is a continuous local martingale. Finally, for a given sequence $\left(x_{n}\right)$ in some convex set, we say that a sequence $\left(\tilde{x}_{n}\right)$ is in the asymptotic convex hull of $\left(x_{n}\right)$ if $\tilde{x}_{n} \in \operatorname{conv}\left\{x_{n}, x_{n+1}, \ldots\right\}$, for all $n \in \mathbb{N}$.

\section{Minimal supersolutions of BSDEs Under Delta And Gamma Constraints}

\subsection{Definitions}

Throughout this work, a generator is a jointly measurable function $g$ from $\Omega \times[0, T] \times \mathbb{R} \times \mathbb{R}^{1 \times d} \times \mathbb{R}^{1 \times d} \times \mathbb{R}^{d \times d}$ to $\mathbb{R} \cup\{+\infty\}$ where $\Omega \times[0, T]$ is endowed with the progressive $\sigma$-field. A control $Z \in \mathcal{L}$ with initial value $z \in \mathbb{R}^{1 \times d}$ is said to have the decomposition $(\Delta, \Gamma)$ if it is of the form $Z=z+\int \Delta \mathrm{d} u+\int \Gamma \mathrm{d} W$, for progressively measurable $(\Delta, \Gamma)$ taking values in $\mathbb{R}^{1 \times d} \times \mathbb{R}^{d \times d 4}$. A control is said to be admissible if the continuous local martingale $\int Z \mathrm{~d} W$ is a supermartingale. Let us collect all these processes in the set $\Theta$ defined by

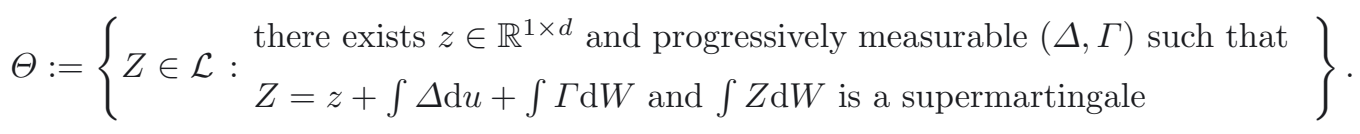

Whenever we want to stress the dependence of controls on a fixed initial value $z \in \mathbb{R}^{1 \times d}$, we make use of the set $\Theta(z):=\left\{Z \in \Theta: Z_{0}=z\right\}$. Given a generator $g$ and a terminal condition $\xi \in L^{0}$, a pair $(Y, Z) \in \mathcal{S} \times \Theta$ is a supersolution of a BSDE under gamma and delta constraints if, for $0 \leq s \leq t \leq T$, it holds

$$
Y_{s}-\int_{s}^{t} g_{u}\left(Y_{u}, Z_{u}, \Delta_{u}, \Gamma_{u}\right) \mathrm{d} u+\int_{s}^{t} Z_{u} \mathrm{~d} W_{u} \geq Y_{t} \quad \text { and } \quad Y_{T} \geq \xi
$$

\footnotetext{
${ }^{4}$ In order to be compatible with the dimension of $Z$, actually the transpose $\left(\int \Gamma \mathrm{d} W\right)^{T}$ of $\int \Gamma \mathrm{d} W$ needs to be considered. However, we suppress this operation for the remainder in order to keep the notation simple.
} 
For a supersolution $(Y, Z)$, we call $Y$ the value process and $Z$ its corresponding control process ${ }^{5}$. Given $z \in \mathbb{R}^{1 \times d}$, we are now interested in the set

$$
\mathcal{A}(\xi, g, z):=\{(Y, Z) \in \mathcal{S} \times \Theta(z):(3.1) \text { holds }\} .
$$

Throughout this paper a generator $g$ is said to be

(LSC) if $(y, z, \delta, \gamma) \mapsto g(y, z, \delta, \gamma)$ is lower semicontinuous.

(POS) positive, if $g(y, z, \delta, \gamma) \geq 0$, for all $(y, z, \delta, \gamma) \in \mathbb{R} \times \mathbb{R}^{1 \times d} \times \mathbb{R}^{1 \times d} \times \mathbb{R}^{d \times d}$.

(CON) convex, if $(y, z, \delta, \gamma) \mapsto g(y, z, \delta, \gamma)$ is jointly convex.

(DGC) delta- and gamma-compatible, if there exist $c_{1} \in \mathbb{R}$ and $c_{2}>0$ such that, for all $(\delta, \gamma) \in \mathbb{R}^{1 \times d} \times \mathbb{R}^{d \times d}$,

$$
g(y, z, \delta, \gamma) \geq c_{1}+c_{2}\left(|\delta|^{2}+|\gamma|^{2}\right)
$$

holds for all $(y, z) \in \mathbb{R} \times \mathbb{R}^{1 \times d}$.

\section{Remark 3.1.}

(i) Note that (DGC) reflects a penalization of rapid changes in control values. In contrast to [3] or [4], where the single decomposition parts $\Delta$ and $\Gamma$ were demanded to satisfy certain boundedness, continuity or growth properties, we embed this in (DGC) so that suitable $\mathcal{L}^{2}$-bounds emerge naturally from the problem (3.1).

(ii) An example of a generator that excludes values of $\Gamma$ exceeding a certain level by penalization and fits into our setting is given by

$$
g(y, z, \delta, \gamma)= \begin{cases}\tilde{g}(y, z, \delta) & \text { if }|\gamma| \leq M \\ +\infty & \text { else, }\end{cases}
$$

where $M>0$ and $\tilde{g}$ is any positive, jointly convex and lower semicontinuous generator satisfying $\tilde{g}(y, z, \delta) \geq$ $c_{1}+c_{2}|\delta|^{2}$ for constants $c_{1} \in \mathbb{R}$ and $c_{2}>0$. This particular choice of $g$ is closely related to the kind of gamma constraints studied in [3].

(iii) Setting the generator $g(\cdot, z, \cdot, \cdot)$ equal to $+\infty$ outside a desired subset of $\mathbb{R}^{1 \times d}$ shows for instance that our framework is flexible enough to comprise shortselling constraints.

\subsection{General properties}

The proof of the ensuing Lemma 3.2 can be found in ([9], Lem. 3.2).

Lemma 3.2. Let $g$ be a generator satisfying (POS). Assume further that $\mathcal{A}(\xi, g) \neq \emptyset$ and that for the terminal condition $\xi$ it holds $\xi^{-} \in L^{1}$. Then $\xi \in L^{1}$ and, for any $(Y, Z) \in \mathcal{A}(\xi, g)$, the control $Z$ is unique and the value process $Y$ is a supermartingale such that $Y_{t} \geq E\left[\xi \mid \mathcal{F}_{t}\right]$. Moreover, the unique canonical decomposition of $Y$ is given by

$$
Y=Y_{0}+M-A,
$$

where $M=\int Z \mathrm{~d} W$ and $A$ is an increasing, predictable, càdlàg process with $A_{0}=0$.

The joint convexity of the generator $g$ immediately yields the following lemma.

Lemma 3.3. Let $g$ be a generator satisfying (CON). Then, for each $z \in \mathbb{R}^{1 \times d}$, the set $\mathcal{A}(\xi, g, z)$ is convex. Furthermore, from $\mathcal{A}\left(\xi^{1}, g, z^{1}\right) \neq \emptyset$ and $\mathcal{A}\left(\xi^{2}, g, z^{2}\right) \neq \emptyset$ follows $\mathcal{A}\left(\xi^{\lambda}, g, z^{\lambda}\right) \neq \emptyset$, for $z^{\lambda}:=\lambda z^{1}+(1-\lambda) z^{2}$ and $\xi^{\lambda}:=\lambda \xi^{1}+(1-\lambda) \xi^{2}$ where $\lambda \in[0,1]$.

\footnotetext{
${ }^{5}$ Note that the formulation in (3.1) is equivalent to the existence of a càdlàg increasing process $K$, with $K_{0}=0$, such that $Y_{t}=\xi+\int_{t}^{T} g_{u}\left(Y_{u}, Z_{u}, \Delta_{u}, \Gamma_{u}\right) \mathrm{d} u+\left(K_{T}-K_{t}\right)-\int_{t}^{T} Z_{u} \mathrm{~d} W_{u}$ for all $t \in[0, T]$, see for example $[11,19]$.
} 
Proof. The first assertion is a direct implication of (CON). As to the latter, it follows from (CON) that $\lambda\left(Y^{1}, Z^{1}\right)+(1-\lambda)\left(Y^{2}, Z^{2}\right) \in \mathcal{A}\left(\xi^{\lambda}, g, z^{\lambda}\right)$ whenever $\left(Y^{1}, Z^{1}\right)$ and $\left(Y^{2}, Z^{2}\right)$ belong to $\mathcal{A}\left(\xi^{1}, g, z^{1}\right)$ and $\mathcal{A}\left(\xi^{2}, g, z^{2}\right)$, respectively.

For the proof of our main existence theorem we will need an auxiliary result concerning the stability of the set $\Theta(z)$ under convergence in $\mathcal{L}^{2}$, given that the decomposition parts can be uniformly bounded in $\mathcal{L}^{2}$.

Lemma 3.4. For any $M>0$ and $z \in \mathbb{R}^{1 \times d}$, the set

$$
\Theta_{M}(z)=\left\{Z \in \Theta(z): \max \left\{\|\Delta\|_{\mathcal{L}^{2}},\|\Gamma\|_{\mathcal{L}^{2}}\right\} \leq M\right\}
$$

is closed under convergence in $\mathcal{L}^{2}$. If a sequence $\left(Z^{n}\right) \subset \Theta_{M}(z)$ with $Z^{n}=z+\int \Delta^{n} \mathrm{~d} t+\int \Gamma^{n} \mathrm{~d} W$ converges in $\mathcal{L}^{2}$ to some $Z=z+\int \Delta \mathrm{d} t+\int \Gamma \mathrm{d} W$, then there is a sequence $\left(\left(\tilde{\Delta}^{n}, \tilde{\Gamma}^{n}\right)\right)$ in the asymptotic convex hull of $\left(\left(\Delta^{n}, \Gamma^{n}\right)\right)$ converging in $\mathcal{L}^{2} \times \mathcal{L}^{2}$ to $(\Delta, \Gamma)$.

Proof. First observe that for $Z \in \Theta(z)$ we have

$$
\left|Z_{t}\right|^{2} \leq 4\left(|z|^{2}+\int_{0}^{t}\left|\Delta_{s}\right|^{2} \mathrm{~d} s+\left|\int_{0}^{t} \Gamma_{s} \mathrm{~d} W_{s}\right|^{2}\right) .
$$

Hence, for $Z \in \Theta_{M}(z)$, this in turn yields $E\left[\left|Z_{t}\right|^{2}\right] \leq 4\left(|z|^{2}+\|\Delta\|_{\mathcal{L}^{2}}^{2}+\|\Gamma\|_{\mathcal{L}^{2}}^{2}\right) \leq 4\left(|z|^{2}+2 M^{2}\right):=C<\infty$, and hence $\Theta_{M}(z)$ is a bounded subset of $\mathcal{L}^{2}$, since by Fubini's theorem we obtain that $\|Z\|_{\mathcal{L}^{2}} \leq \sqrt{T C}$. Consider a sequence $Z^{n}=z+\int \Delta^{n} \mathrm{~d} u+\int \Gamma^{n} \mathrm{~d} W$ in $\Theta_{M}(z)$ converging in $\mathcal{L}^{2}$ to some process $Z$. Since $\left(\left(\Delta^{n}, \Gamma^{n}\right)\right)$ are bounded in $\mathcal{L}^{2} \times \mathcal{L}^{2}$, we can find a sequence $\left(\tilde{\Delta}^{n}, \tilde{\Gamma}^{n}\right) \in \operatorname{conv}\left\{\left(\Delta^{n}, \Gamma^{n}\right),\left(\Delta^{n+1}, \Gamma^{n+1}\right), \ldots\right\}$ converging in $\mathcal{L}^{2} \times \mathcal{L}^{2}$ to some $(\Delta, \Gamma) \in \mathcal{L}^{2} \times \mathcal{L}^{2}$. Furthermore, it holds that $\|\Delta\|_{\mathcal{L}^{2}} \vee\|\Gamma\|_{\mathcal{L}^{2}} \leq M$. Let us denote by $\left(\tilde{Z}^{n}\right)$ the respective sequence in the asymptotic convex hull of $\left(Z^{n}\right)$. From Jensen's inequality we deduce that

$$
E\left[\int_{0}^{T}\left|\int_{0}^{t}\left(\tilde{\Delta}_{s}^{n}-\Delta_{s}\right) \mathrm{d} s\right|^{2} \mathrm{~d} t\right] \leq T E\left[\int_{0}^{T}\left|\tilde{\Delta}_{s}^{n}-\Delta_{s}\right|^{2} \mathrm{~d} s\right] \rightarrow 0,
$$

and thus $\left(\int \tilde{\Delta}^{n} \mathrm{~d} s\right)$ converges to $\int \Delta \mathrm{d} s$ in $\mathcal{L}^{2}$. Applying Fubini's theorem and using the Itô isometry yield that

$$
E\left[\int_{0}^{T}\left|\int_{0}^{t} \tilde{\Gamma}_{s}^{n} \mathrm{~d} W_{s}-\int_{0}^{t} \Gamma_{s} \mathrm{~d} W_{s}\right|^{2} \mathrm{~d} t\right] \leq T E\left[\int_{0}^{T}\left|\tilde{\Gamma}_{s}^{n}-\Gamma_{s}\right|^{2} \mathrm{~d} s\right],
$$

where the term on the right-hand side tends to zero by means of the $\mathcal{L}^{2}$-convergence of $\left(\tilde{\Gamma}^{n}\right)$ to $\Gamma$. Hence, $\left(\int \tilde{\Gamma}^{n} \mathrm{~d} W\right)$ converges to $\int \Gamma \mathrm{d} W$ in $\mathcal{L}^{2}$. $\left(\tilde{Z}^{n}\right)$ inheriting the $\mathcal{L}^{2}$-convergence to $Z$ from $\left(Z^{n}\right)$ together with the $P \otimes \mathrm{d} t$-uniqueness of $\mathcal{L}^{2}$-limits finally allows us to write the process $Z$ as $Z=z+\int \Delta \mathrm{d} s+\int \Gamma \mathrm{d} W$, we are done.

Lemma 3.4 yields the following compactness result.

Lemma 3.5. Assume that $\mathcal{A}(\xi, g, z)$ is non-empty for some $z \in \mathbb{R}^{1 \times d}$. Let $\xi^{-}$be in $L^{1}$ and $g$ satisfy (POS), $(\mathrm{CON})$ and $(\mathrm{DGC})$. Then, for any sequence $\left(\left(Y^{n}, Z^{n}\right)\right) \subset \mathcal{A}(\xi, g, z)$ of supersolutions satisfying $\sup _{n} Y_{0}^{n}<\infty$, the following holds: There is a sequence $\left(\tilde{Z}^{n}\right)$ in the asymptotic convex hull of $\left(Z^{n}\right)$ that converges in $\mathcal{L}^{2}$ to some process $\hat{Z} \in \Theta(z)$.

Proof.

Step 1: Existence of $\left(\left(\tilde{Y}^{n}, \tilde{Z}^{n}\right)\right)$. $\mathcal{L}^{2}$-convergence of $\left(\tilde{Z}^{n}\right)$ to $\hat{Z}$. First observe that (3.1) and the supermartingale property of all $\int Z^{n} \mathrm{~d} W$ imply that

$$
E\left[\int_{0}^{T} g_{t}\left(Y_{t}^{n}, Z_{t}^{n}, \Delta_{t}^{n}, \Gamma_{t}^{n}\right) \mathrm{d} t\right] \leq Y_{0}^{n}+E\left[\xi^{-}\right] \leq C+E\left[\xi^{-}\right]<\infty,
$$


where we put $C:=\sup _{n} Y_{0}^{n}$. Now, using (3.3) together with (DGC) we estimate

$$
\begin{aligned}
\left\|\Delta^{n}\right\|_{\mathcal{L}^{2}}^{2}+\left\|\Gamma^{n}\right\|_{\mathcal{L}^{2}}^{2} & =E\left[\int_{0}^{T}\left|\Delta_{t}^{n}\right|^{2} \mathrm{~d} t\right]+E\left[\int_{0}^{T}\left|\Gamma_{t}^{n}\right|^{2} \mathrm{~d} t\right] \\
& \leq \frac{1}{c_{2}} E\left[\int_{0}^{T} g_{t}\left(Y_{t}^{n}, Z_{t}^{n}, \Delta_{t}^{n}, \Gamma_{t}^{n}\right) \mathrm{d} t\right]-\frac{c_{1}}{c_{2}} T \leq \frac{1}{c_{2}}\left(C+E\left[\xi^{-}\right]-c_{1} T\right)<\infty .
\end{aligned}
$$

Since the right-hand above is independent of $n$, we obtain that $\left(Z^{n}\right) \subset \Theta_{M}(z)$ with $M:=\left[\frac{1}{c_{2}}\left(C+E\left[\xi^{-}\right]-c_{1} T\right)\right]^{\frac{1}{2}}$ and the arguments within the proof of Lemma 3.4 show that the sequence $\left(Z^{n}\right)$ is uniformly bounded in $\mathcal{L}^{2}$. This in turn guarantees the existence of a sequence $\left(\tilde{Z}^{n}\right)$ in the asymptotic convex hull of $\left(Z^{n}\right)$ that converges to some process $\hat{Z}$ in $\mathcal{L}^{2}$ and, up to a subsequence, $P \otimes \mathrm{d} t$-almost everywhere.

Step 2: The process $\hat{Z}$ belongs to $\Theta(z)$. The sequence $\left(\left(\tilde{Y}^{n}, \tilde{Z}^{n}\right)\right)$ lies in $\mathcal{A}(\xi, g, z)$, due to (CON). Moreover, the linearity of the integrals within the Itô decompositions of $\left(Z^{n}\right)$ yields that $\tilde{Z}^{n}=z+\int \tilde{\Delta}^{n} \mathrm{~d} u+\int \tilde{\Gamma}^{n} \mathrm{~d} W$ where $\left(\left(\tilde{\Delta}^{n}, \tilde{\Gamma}^{n}\right)\right)$ denotes the corresponding convex combinations of the decomposition parts. In addition, $\left(\left(\tilde{\Delta}^{n}, \tilde{\Gamma}^{n}\right)\right)$ inherits the uniform bound from $\left(\left(\Delta^{n}, \Gamma^{n}\right)\right)$, that is $\max \left\{\sup _{n}\left\|\tilde{\Delta}^{n}\right\|_{\mathcal{L}^{2}}, \sup _{n}\left\|\tilde{\Gamma}^{n}\right\|_{\mathcal{L}^{2}}\right\} \leq M$. Hence, Lemma 3.4 ensures that $\hat{Z}$ is of the form

$$
\hat{Z}=z+\int \hat{\Delta} \mathrm{d} u+\int \hat{\Gamma} \mathrm{d} W
$$

with suitable $\mathcal{L}^{2}$-convergence of the decomposition parts by possibly passing to yet another subsequence in the respective asymptotic convex hull. This finishes the proof.

\subsection{Minimality under constraints}

Within the current setup of admissible controls constrained to follow certain dynamics, we are interested in supersolutions $(\hat{Y}, \hat{Z}) \in \mathcal{A}(\xi, g, z)$ minimal at time zero, that is $\hat{Y}_{0} \leq Y_{0}$ for all $(Y, Z) \in \mathcal{A}(\xi, g, z)$. In the remainder of this work, a major role is thus played by the operator

$$
\mathcal{E}_{0}^{g}(\xi, z):=\inf \left\{Y_{0}:(Y, Z) \in \mathcal{A}(\xi, g, z)\right\},
$$

since any $(\hat{Y}, \hat{Z})$ satisfying $\hat{Y}_{0}=\mathcal{E}_{0}^{g}(\xi, z)$ naturally exhibits the property of being minimal at time zero. Note that the definition of a supersolution directly yields that $\mathcal{A}\left(\xi^{1}, g, z\right) \subseteq \mathcal{A}\left(\xi^{2}, g, z\right)$ whenever $\xi^{1} \geq \xi^{2}$. Hence, we immediately obtain monotonicity of the operator $\mathcal{E}_{0}(\cdot, z)$, that is $\xi^{1} \geq \xi^{2}$ implies $\mathcal{E}_{0}^{g}\left(\xi^{1}, z\right) \geq \mathcal{E}_{0}^{g}\left(\xi^{2}, z\right)$. The ensuing Theorem 3.7 provides existence of supersolutions minimal at time zero making use of the fact that the set $\left\{Y_{0}:(Y, Z) \in \mathcal{A}(\xi, g, z)\right\}$ is directed downwards. Parts of it rely on a version of Helly's theorem which we state here for the sake of completeness. In order to keep this work self-contained, we include the proof given in ([12], Lem. 1.25).

Lemma 3.6. Let $\left(A^{n}\right)$ be a sequence of increasing positive processes such that the sequence $\left(A_{T}^{n}\right)$ is bounded in $L^{1}$. Then, there is a sequence $\left(\tilde{A}^{n}\right)$ in the asymptotic convex hull of $\left(A^{n}\right)$ and an increasing positive integrable process $\tilde{A}$ such that

$$
\lim _{n \rightarrow \infty} \tilde{A}_{t}^{n}=\tilde{A}_{t}, \quad \text { for all } t \in[0, T], P \text {-almost surely. }
$$

Proof. Let $\left(t_{j}\right)$ be a sequence running through $I:=([0, T] \cap \mathbb{Q}) \cup\{T\}$. Since $\left(A_{t_{1}}^{n}\right)$ is an $L^{1}$-bounded sequence of positive random variables, due to $\left([7]\right.$, Lem. A1.1) there exists a sequence $\left(\tilde{A}^{1, k}\right)$ in the asymptotic convex hull of $\left(A^{n}\right)$ and a random variable $\tilde{A}_{t_{1}}$ such that $\left(\tilde{A}_{t_{1}}^{1, k}\right)$ converges $P$-almost surely to $\tilde{A}_{t_{1}}$. Moreover, Fatou's lemma yields $\tilde{A}_{t_{1}} \in L^{1}$. Let $\left(\tilde{A}^{2, k}\right)$ be a sequence in the asymptotic convex hull of $\left(\tilde{A}^{1, k}\right)$ such that $\left(\tilde{A}_{t_{2}}^{2, k}\right)$ converges $P$-almost surely to $\tilde{A}_{t_{2}} \in L^{1}$ and so on. Then, for $s \in I$, it holds $\tilde{A}_{s}^{k, k} \rightarrow \tilde{A}_{s}$ on a set $\hat{\Omega} \subset \Omega$ satisfying $P(\hat{\Omega})=1$. The process $\tilde{A}$ is positive, increasing and integrable on $I$. Thus we may define

$$
\hat{A}_{t}:=\lim _{r \downarrow t, r \in I} \tilde{A}_{r}, \quad t \in[0, T), \quad \hat{A}_{T}:=\tilde{A}_{T} .
$$


We now show that $\left(\tilde{A}^{k, k}\right)$, henceforth named $\left(\tilde{A}^{k}\right)$, converges $P$-almost surely on the continuity points of $\hat{A}$. To this end, fix $\omega \in \hat{\Omega}$ and a continuity point $t \in[0, T)$ of $\hat{A}(\omega)$. We show that $\left(\tilde{A}_{t}^{k}(\omega)\right)$ is a Cauchy sequence in $\mathbb{R}$. Fix $\varepsilon>0$ and set $\delta=\frac{\varepsilon}{11}$. Since $t$ is a continuity point of $\hat{A}(\omega)$, we may choose $p_{1}, p_{2} \in I$ such that $p_{1}<t<p_{2}$ and $\hat{A}_{p_{1}}(\omega)-\hat{A}_{p_{2}}(\omega)<\delta$. By definition of $\hat{A}$, we may choose $r_{1}, r_{2} \in I$ such that $p_{1}<r_{1}<t<p_{2}<r_{2}$ and $\left|\hat{A}_{p_{1}}(\omega)-\tilde{A}_{r_{1}}(\omega)\right|<\delta$ and $\left|\hat{A}_{p_{2}}(\omega)-\tilde{A}_{r_{2}}(\omega)\right|<\delta$. Now choose $N \in \mathbb{N}$ such that $\left|\tilde{A}_{r_{1}}^{m}(\omega)-\tilde{A}_{r_{1}}^{n}(\omega)\right|<\delta$, for all $m, n \in \mathbb{N}$ with $m, n \geq N$, and $\left|\tilde{A}_{r_{2}}^{j}(\omega)-\tilde{A}_{r_{2}}(\omega)\right|<\delta$ and $\left|\tilde{A}_{r_{1}}(\omega)-\tilde{A}_{r_{1}}^{j}(\omega)\right|<\delta$ for $j=m, n$. We estimate

$$
\left|\tilde{A}_{t}^{m}(\omega)-\tilde{A}_{t}^{n}(\omega)\right| \leq\left|\tilde{A}_{t}^{m}(\omega)-\tilde{A}_{r_{1}}^{m}(\omega)\right|+\left|\tilde{A}_{r_{1}}^{m}(\omega)-\tilde{A}_{r_{1}}^{n}(\omega)\right|+\left|\tilde{A}_{r_{1}}^{n}(\omega)-\tilde{A}_{t}^{n}(\omega)\right| .
$$

For the first and the third term on the right hand side, since $\tilde{A}^{m}$ and $\tilde{A}^{n}$ are increasing, we deduce that $\left|\tilde{A}_{t}^{m}(\omega)-\tilde{A}_{r_{1}}^{m}(\omega)\right| \leq\left|\tilde{A}_{r_{2}}^{m}(\omega)-\tilde{A}_{r_{1}}^{m}(\omega)\right|$ and $\left|\tilde{A}_{t}^{n}(\omega)-\tilde{A}_{r_{1}}^{n}(\omega)\right| \leq\left|\tilde{A}_{r_{2}}^{n}(\omega)-\tilde{A}_{r_{1}}^{n}(\omega)\right|$. Furthermore,

$$
\begin{aligned}
\left|\tilde{A}_{r_{2}}^{j}(\omega)-\tilde{A}_{r_{1}}^{j}(\omega)\right| \leq\left|\tilde{A}_{r_{2}}^{j}(\omega)-\tilde{A}_{r_{2}}(\omega)\right|+\left|\tilde{A}_{r_{2}}(\omega)-\hat{A}_{p_{2}}(\omega)\right| \\
\\
+\left|\hat{A}_{p_{2}}(\omega)-\hat{A}_{p_{1}}(\omega)\right|+\left|\hat{A}_{p_{1}}(\omega)-\tilde{A}_{r_{1}}(\omega)\right|+\left|\tilde{A}_{r_{1}}(\omega)-\tilde{A}_{r_{1}}^{j}(\omega)\right|,
\end{aligned}
$$

for $j=m, n$. Combining the previous inequalities yields $\left|\tilde{A}_{t}^{m}(\omega)-\tilde{A}_{t}^{n}(\omega)\right| \leq \varepsilon$, for all $m, n \geq N$. Hence, $\left(\tilde{A}^{k}(\omega)\right)$ converges for all continuity points $t \in[0, T)$ of $\hat{A}(\omega)$, for all $\omega \in \hat{\Omega}$. We denote the limit by $\tilde{A}$.

It remains to be shown that $\left(\tilde{A}^{k}\right)$ also converges for the discontinuity points of $\hat{A}$. To this end, note that $\hat{A}$ is càdlàg and adapted to our filtration which fulfills the usual conditions. By a well-known result (see for example [15], Prop. 1.2.26), this implies that the jumps of $\hat{A}$ may be exhausted by a sequence of stopping times $\left(\rho^{j}\right)$. Applying once more ([7], Lem. A1.1) iteratively on the sequences $\left(\tilde{A}_{\rho^{j}}^{k}\right)_{k \in \mathbb{N}}, j=1,2,3 \ldots$, and diagonalizing yields the result.

Theorem 3.7. Assume that $\mathcal{A}(\xi, g, z) \neq \emptyset$ for some $\xi^{-} \in L^{1}$ and $z \in \mathbb{R}^{1 \times d}$ and let $g$ satisfy (LSC), (POS), (CON) and (DGC). Then, the set $\left\{(\hat{Y}, \hat{Z}) \in \mathcal{A}(\xi, g, z): \hat{Y}_{0}=\mathcal{E}_{0}^{g}(\xi, z)\right\}$ is non-empty.

Proof.

Step 1: The candidate control $\hat{Z}$. We extract a sequence $\left(\left(Y^{n}, Z^{n}\right)\right) \subset \mathcal{A}(\xi, g, z)$ such that

$$
\lim _{n \rightarrow \infty} Y_{0}^{n}=\mathcal{E}_{0}^{g}(\xi, z) .
$$

Because $\sup _{n} Y_{0}^{n} \leq Y_{0}^{1}<\infty$, Lemma 3.5 assures the existence of a sequence $\left(\tilde{Z}^{n}\right)$ in the asymptotic convex hull of $\left(Z^{n}\right)$ that converges in $\mathcal{L}^{2}$ to some admissible process $\hat{Z} \in \Theta(z)$, including $\mathcal{L}^{2}$-convergence of the corresponding decomposition parts. In particular, we obtain that

$$
\int_{0}^{t} \tilde{Z}_{u}^{n} \mathrm{~d} W_{u} \underset{n \rightarrow \infty}{\longrightarrow} \int_{0}^{t} \hat{Z}_{u} \mathrm{~d} W_{u}, \quad \text { for all } t \in[0, T], P \text {-almost surely. }
$$

Moreover, up to a subsequence, $\left(\left(\tilde{Z}^{n}, \tilde{\Delta}^{n}, \tilde{\Gamma}^{n}\right)\right)$ converges $P \otimes \mathrm{d} t$-almost everywhere towards $(\hat{Z}, \hat{\Delta}, \hat{\Gamma})$.

Step 2: The candidate value process $\hat{Y}$. If we denote by $\left(\tilde{Y}^{n}\right)$ the sequence in the asymptotic convex hull of $\left(Y^{n}\right)$ corresponding to $\left(\tilde{Z}^{n}\right)$, then all $\left(\tilde{Y}^{n}, \tilde{Z}^{n}\right)$ satisfy $(3.1)$ due to (CON). Let $\tilde{A}^{n}$ denote the increasing, predicable process of finite variation stemming from the decomposition of $\tilde{Y}^{n}=\tilde{Y}_{0}^{n}+\tilde{M}^{n}-\tilde{A}^{n}$ given in Lemma 3.2. Since $\left(\tilde{Z}^{n}\right)$ is uniformly bounded in $\mathcal{L}^{2}$ and thus all $\int \tilde{Z}^{n} \mathrm{~d} W$ are true martingales, and $g$ satisfies (POS), the decomposition (3.2) yields

$$
E\left[\tilde{A}_{T}^{n}\right] \leq Y_{0}^{1}+E\left[\xi^{-}\right]<\infty
$$

as we assumed $\xi^{-}$to be an element of $L^{1}$. Now a version of Helly's theorem, see Lemma 3.6, yields the existence of a sequence in the asymptotic convex hull of $\left(\tilde{A}^{n}\right)$, again denoted by the previous expression, and of an increasing positive integrable process $\tilde{A}$ such that $\lim _{n \rightarrow \infty} \tilde{A}_{t}^{n}=\tilde{A}_{t}$, for all $t \in[0, T], P$-almost surely. We pass 
to the corresponding sequence on the side of $\left(\tilde{Y}^{n}\right)$ and $\left(\tilde{Z}^{n}\right)$, define the process $\tilde{Y}$ pointwise for all $t \in[0, T]$ by $\tilde{Y}_{t}:=\lim _{n \rightarrow \infty} \tilde{Y}_{t}^{n}=\mathcal{E}_{0}^{g}(\xi, z)+\int_{0}^{t} \hat{Z}_{u} \mathrm{~d} W_{u}-\tilde{A}_{t}$, and observe that it fulfills $\tilde{Y}_{0}=\mathcal{E}_{0}^{g}(\xi, z)$ by construction. However, since $\tilde{Y}$ is not necessarily càdlàg, we define our candidate value process $\hat{Y}$ by $\hat{Y}_{t}:=\lim _{s \downarrow t, s \in \mathbb{Q}} \tilde{Y}_{s}$, for all $t \in[0, T)$ and $\hat{Y}_{T}:=\xi$. The continuity of $\int \hat{Z} \mathrm{~d} W$ yields that

$$
\hat{Y}_{t}=\mathcal{E}_{0}^{g}(\xi, z)+\int_{0}^{t} \hat{Z}_{u} \mathrm{~d} W_{u}-\lim _{s \downarrow t, s \in \mathbb{Q}} \tilde{A}_{s} .
$$

Since jump times of càdlàg processes ${ }^{6}$ can be exhausted by a sequence of stopping times $\left(\sigma_{j}\right) \subset \mathcal{T}$, compare ([15], Prop. 1.2.26), which coincide with the jump times of $\tilde{A}$, we conclude that

$$
\hat{Y}=\tilde{Y}, \quad P \otimes \mathrm{d} t \text {-almost everywhere. }
$$

Furthermore, $\tilde{A}$ increasing implies that $\hat{A}_{t}:=\lim _{s \downarrow t, s \in \mathbb{Q}} \tilde{A}_{s} \geq \tilde{A}_{t}$, for all $t \in[0, T]$ which, together with (3.6), in turn yields that

$$
\hat{Y}_{t} \leq \tilde{Y}_{t}, \quad \text { for all } t \in[0, T] .
$$

Given that $(\hat{Y}, \hat{Z})$ satisfies (3.1), we could conclude that $(\hat{Y}, \hat{Z}) \in \mathcal{A}(\xi, g, z)$ and thus $\hat{Y}_{0} \geq \mathcal{E}_{0}^{g}(\xi, z)=\tilde{Y}_{0}$ which, combined with (3.8), would imply $\hat{Y}_{0}=\mathcal{E}_{0}^{g}(\xi, z)$ and thereby finish the proof.

Step 3: Verification. As to the remaining verification, we deduce from (3.7) the existence of a set $A \in \mathcal{F}_{T}$, $P(A)=1$ with the following property. For all $\omega \in A$, there exists a Lebesgue measurable set $\mathcal{I}(\omega) \subset[0, T]$ of measure $T$ such that $\tilde{Y}_{t}^{n}(\omega) \longrightarrow \hat{Y}_{t}(\omega)$, for all $t \in \mathcal{I}(\omega)$. We suppress the dependence of $\mathcal{I}$ on $\omega$ and recall however that in the following $s$ and $t$ may depend on $\omega$. For $s, t \in \mathcal{I}$ with $s \leq t$ holds

$$
\hat{Y}_{s}-\int_{s}^{t} g_{u}\left(\hat{Y}_{u}, \hat{Z}_{u}, \hat{\Delta}_{u}, \hat{\Gamma}_{u}\right) \mathrm{d} u+\int_{s}^{t} \hat{Z}_{u} \mathrm{~d} W_{u} \geq \underset{n}{\lim \sup }\left(\tilde{Y}_{s}^{n}-\int_{s}^{t} g_{u}\left(\tilde{Y}_{u}^{n}, \tilde{Z}_{u}^{n}, \tilde{\Delta}_{u}^{n}, \tilde{\Gamma}_{u}^{n}\right) \mathrm{d} u+\int_{s}^{t} \tilde{Z}_{u}^{n} \mathrm{~d} W_{u}\right)
$$

by means of (3.5), the $P \otimes \mathrm{d} t$-almost-everywhere convergence of $\left(\left(\tilde{Y}^{n}, \tilde{Z}^{n}, \tilde{\Delta}^{n}, \tilde{\Gamma}^{n}\right)\right)$ towards $(\hat{Y}, \hat{Z}, \hat{\Delta}, \hat{\Gamma})$, the property (LSC) and Fatou's lemma. Using $\left(\left(\tilde{Y}^{n}, \tilde{Z}^{n}\right)\right) \subset \mathcal{A}(\xi, g, z)$, for all $n \in \mathbb{N}$, (3.9) can be further estimated by

$$
\hat{Y}_{s}-\int_{s}^{t} g_{u}\left(\hat{Y}_{u}, \hat{Z}_{u}, \hat{\Delta}_{u}, \hat{\Gamma}_{u}\right) \mathrm{d} u+\int_{s}^{t} \hat{Z}_{u} \mathrm{~d} W_{u} \geq \limsup _{n} \tilde{Y}_{t}^{n}=\hat{Y}_{t}
$$

Whenever $s, t \in \mathcal{I}^{c}$ with $s \leq t$, we approximate both times from the right by sequences $\left(s^{n}\right) \subset \mathcal{I}$ and $\left(t^{n}\right) \subset \mathcal{I}$, respectively, such that $s^{n} \leq t^{n}$. Since (3.10) holds for all $s^{n}$ and $t^{n}$, the claim follows from the right-continuity of $\hat{Y}$ and the continuity of all appearing integrals, which finally concludes the proof.

Convexity of the mapping $(\xi, z) \mapsto \mathcal{E}_{0}^{g}(\xi, z)$ is provided by the following lemma.

Lemma 3.8. Under the assumptions of Theorem 3.7 , the operator $\mathcal{E}_{0}^{g}(\cdot, \cdot)$ is jointly convex.

Proof. For $z^{1}, z^{2} \in \mathbb{R}^{1 \times d}$ and $\xi^{1}, \xi^{2} \in L^{0}$, the negative parts of which are integrable, assume that $\mathcal{A}\left(\xi^{1}, g, z^{1}\right) \neq \emptyset$ and $\mathcal{A}\left(\xi^{2}, g, z^{2}\right) \neq \emptyset$, as otherwise convexity trivially holds. For $\lambda \in[0,1]$ we set $z^{\lambda}:=\lambda z^{1}+(1-\lambda) z^{2}$ and $\xi^{\lambda}:=\lambda \xi^{1}+(1-\lambda) \xi^{2}$ so that Lemma 3.3 implies $\mathcal{A}\left(\xi^{\lambda}, g, z^{\lambda}\right) \neq \emptyset$. By Theorem 3.7, there exist $\left(Y^{1}, Z^{1}\right)$ and $\left(Y^{2}, Z^{2}\right)$ in $\mathcal{A}\left(\xi^{1}, g, z^{1}\right)$ and $\mathcal{A}\left(\xi^{2}, g, z^{2}\right)$, respectively, such that $Y_{0}^{1}=\mathcal{E}_{0}^{g}\left(\xi^{1}, z^{1}\right)$ and $Y_{0}^{2}=\mathcal{E}_{0}^{g}\left(\xi^{2}, z^{2}\right)$. Since $(\bar{Y}, \bar{Z}):=\lambda\left(Y^{1}, Z^{1}\right)+(1-\lambda)\left(Y^{2}, Z^{2}\right)$ is an element of $\mathcal{A}\left(\xi^{\lambda}, g, z^{\lambda}\right)$ due to (CON), it holds $\mathcal{E}_{0}^{g}\left(\xi^{\lambda}, z^{\lambda}\right) \leq \bar{Y}_{0}$ by definition of the operator $\mathcal{E}_{0}^{g}$.

\footnotetext{
${ }^{6}$ Note that as an increasing process, $\tilde{A}$ is in particular a submartingale and thus its right- and left-hand limits exist, compare ([15], Prop. 1.3.14). Consequently, the process $\lim _{s \downarrow \cdot, s \in \mathbb{Q}} \tilde{A}_{s}$ is càdlàg.
} 


\subsection{Stability results}

Next, we show that the non-linear operator $\xi \mapsto \mathcal{E}_{0}^{g}(\xi, z)$ exhibits stability properties such as monotone convergence, the Fatou property or $L^{1}$-lower semicontinuity. The following theorem establishes monotone convergence and the Fatou property of $\mathcal{E}_{0}^{g}(\cdot, z)$. Similar results in the unconstrained case have been obtained in ([9], Thm. 4.7).

Theorem 3.9. For $z \in \mathbb{R}^{1 \times d}$ and $g$ a generator fulfilling (LSC), (POS), (CON) and (DGC), and ( $\left.\xi_{n}\right)$ a sequence in $L^{0}$ such that $\left(\xi_{n}^{-}\right) \subset L^{1}$, the following holds.

- Monotone convergence: If $\left(\xi_{n}\right)$ is increasing P-almost surely to $\xi \in L^{0}$, then it holds $\lim _{n \rightarrow \infty} \mathcal{E}_{0}^{g}\left(\xi_{n}, z\right)=$ $\mathcal{E}_{0}^{g}(\xi, z)$.

- Fatou's lemma: If $\xi_{n} \geq \eta$, for all $n \in \mathbb{N}$, where $\eta \in L^{1}$, then it holds $\mathcal{E}_{0}^{g}\left(\liminf _{n} \xi_{n}, z\right) \leq \liminf _{n} \mathcal{E}_{0}^{g}\left(\xi_{n}, z\right)$.

Proof.

Monotone convergence: First, note that by monotonicity the limit $\bar{Y}_{0}:=\lim _{n} \mathcal{E}_{0}^{g}\left(\xi_{n}, z\right)$ exists and satisfies $\bar{Y}_{0} \leq \mathcal{E}_{0}^{g}(\xi, z)$. Other than in the trivial case of $+\infty=\bar{Y}_{0} \leq \mathcal{E}_{0}^{g}(\xi, z)$ we have $\mathcal{A}\left(\xi_{n}, g, z\right) \neq \emptyset$, for all $n \in \mathbb{N}$, which, together with $\left(\xi_{n}^{-}\right) \subset L^{1}$ implies $\left(\xi_{n}\right) \subset L^{1}$. Furthermore, Theorem 3.7 yields the existence of supersolutions $\left(Y^{n}, Z^{n}\right) \in \mathcal{A}\left(\xi_{n}, g, z\right)$ fulfilling $Y_{0}^{n}=\mathcal{E}_{0}^{g}\left(\xi_{n}, z\right)$, for all $n \in \mathbb{N}$. In particular, we have that $Y_{0}^{n} \leq \bar{Y}_{0}$ and $\xi_{n}^{-} \leq \xi_{1}^{-}$, for all $n \in \mathbb{N}$. Arguments analogous to the ones used in Lemma 3.5 and the proof of Theorem 3.7 directly translate to the present setting and provide both a candidate control $\hat{Z} \in \Theta(z)$ to which $\left(\tilde{Z}^{n}\right)$ converges and a corresponding $\tilde{Y}_{t}:=\lim _{n} \tilde{Y}_{t}^{n}$, and ensure that $(\hat{Y}, \hat{Z})$ belongs to $\mathcal{A}(\xi, g, z)$, where $\hat{Y}:=\lim _{s \in \mathbb{Q}, s \downarrow} . \tilde{Y}_{s}$ on $[0, T)$ and $\hat{Y}_{T}:=\xi$. In particular, we obtain $\hat{Y}_{0} \leq \tilde{Y}_{0}=\bar{Y}_{0}$. Hence, as $\mathcal{A}(\xi, g, z) \neq \emptyset$ and $\xi^{-} \in L^{1}$, there exists $(Y, Z) \in \mathcal{A}(\xi, g, z)$ such that $Y_{0}=\mathcal{E}_{0}^{g}(\xi, z)$. By minimality of $(Y, Z)$ at time zero, however, this entails $Y_{0} \leq \hat{Y}_{0} \leq \bar{Y}_{0}$ and we conclude that $\lim _{n \rightarrow \infty} \mathcal{E}_{0}^{g}\left(\xi_{n}, z\right)=\mathcal{E}_{0}^{g}(\xi, z)$.

Fatou's lemma: If we define $\zeta_{n}:=\inf _{k \geq n} \xi_{k}$, then $\xi_{k} \geq \eta$ for all $k \in \mathbb{N}$ implies $\zeta_{n} \geq \eta$ for all $n \in \mathbb{N}$ which in turn gives $\left(\zeta_{n}^{-}\right) \subset L^{1}$, and thus the monotone convergence established above can be used exactly as in ([9], Thm. 4.7) to obtain the assertion.

As a consequence of the monotone convergence property we obtain the ensuing theorem providing $L^{1}$-lower semicontinuity of the operator $\mathcal{E}_{0}^{g}(\cdot, z)$. The proof goes along the lines of ([9], Thm. 4.9) and is thus omitted here.

Theorem 3.10. Let $z \in \mathbb{R}^{1 \times d}$ and $g$ be a generator fulfilling (LSC), (POS), (CON) and(DGC). Then $\mathcal{E}_{0}^{g}(\cdot, z)$ is $L^{1}$-lower semicontinuous.

\section{DuAlity under CONSTRAints}

The objective of this section is to construct a solution of constrained BSDEs via duality and, for the case of a quadratic generator, to obtain an explicit form for $\mathcal{E}_{0}^{*}$, the Fenchel-Legendre transform of $\mathcal{E}_{0}^{g}$. Let us assume for the rest of this section that our generator $g$ is independent of $y$, that is $g_{u}(y, z, \delta, \gamma)=g_{u}(z, \delta, \gamma)$, and that it satisfies (LSC), (POS), (CON) and (DGC). Let us further fix some $z \in \mathbb{R}^{1 \times d}$ as initial value of the controls and set $\mathcal{E}_{0}^{g}(\cdot):=\mathcal{E}_{0}^{g}(\cdot, z)$ for the remainder of this section. Whenever we say that the $\operatorname{BSDE}(\xi, g)$ has a solution $(Y, Z)$, we mean that there exists $(Y, Z) \in \mathcal{A}(\xi, g, z)$ such that (3.1) is satisfied with equalities instead of inequalities. Observe that $\mathcal{E}_{0}^{g}(\cdot)$, being convex and $L^{1}$-lower semicontinuous, is in particular $\sigma\left(L^{1}, L^{\infty}\right)$-lower semicontinuous, and thus, by classical duality results admits the Fenchel-Moreau representation

$$
\mathcal{E}_{0}^{g}(\xi)=\sup _{v \in L^{\infty}}\left\{E[v \xi]-\mathcal{E}_{0}^{*}(v)\right\}, \quad \xi \in L^{1},
$$

where for $v \in L^{\infty}$ the convex conjugate is given by

$$
\mathcal{E}_{0}^{*}(v):=\sup _{\xi \in L^{1}}\left\{E[v \xi]-\mathcal{E}_{0}^{g}(\xi)\right\} .
$$


It is proved in the next lemma that the domain of $\mathcal{E}_{0}^{*}$ is concentrated on non-negative $v \in L_{+}^{\infty}$ satisfying $E[v]=1$.

Lemma 4.1. Within the representation (4.1), that is $\mathcal{E}_{0}^{g}(\xi)=\sup _{v \in L^{\infty}}\left\{E[v \xi]-\mathcal{E}_{0}^{*}(v)\right\}$, the supremum might be restricted to those $v \in L_{+}^{\infty}$ satisfying $E[v]=1$.

Proof. First, we assume without loss of generality that $\mathcal{E}_{0}^{g}(0)<+\infty$. Indeed, a slight modification of the argumentation below remains valid using any $\xi \in L^{1}$ such that $\mathcal{E}_{0}^{g}(\xi)<+\infty^{7}$. We show that $\mathcal{E}_{0}^{*}(v)=+\infty$ as soon as $v \in L^{\infty} \backslash L_{+}^{\infty}$ or $E[v] \neq 1$. For $v \in L^{\infty} \backslash L_{+}^{\infty}, L_{+}^{1}$ being the polar of $L_{+}^{\infty}$ yields the existence of $\bar{\xi} \in L_{+}^{1}$ such that $E[v \bar{\xi}]<0$. Monotonicity of $\mathcal{E}_{0}^{g}$ then gives $\mathcal{E}_{0}^{g}(-n \bar{\xi}) \leq \mathcal{E}_{0}^{g}(0)$ for all $n \in \mathbb{N}$. Hence,

$$
\mathcal{E}_{0}^{*}(v) \geq \sup _{n}\left\{n E[-v \bar{\xi}]-\mathcal{E}_{0}^{g}(-n \bar{\xi})\right\} \geq \sup _{n}\{n E[-v \bar{\xi}]\}-\mathcal{E}_{0}^{g}(0)=+\infty .
$$

Furthermore, since the generator does not depend on $y$, the function $\mathcal{E}_{0}^{g}$ is cash additive, compare ([9], Prop. 3.3.5), and we deduce that, for all $n \in \mathbb{N}$ it holds

$$
\mathcal{E}_{0}^{*}(v) \geq E[v n]-\mathcal{E}_{0}^{g}(0)-n=n(E[v]-1)-\mathcal{E}_{0}^{g}(0) .
$$

Thus, if $E[v]>1$, then $\mathcal{E}_{0}^{*}(v)=+\infty$. A reciprocal argument with $\xi=-n$ finally gives $\mathcal{E}_{0}^{*}(v)=+\infty$ whenever $E[v]<1$.

By the previous result, we may use $v \in L_{+}^{\infty}, E[v]=1$, in order to define a measure $Q$ that is absolutely continuous with respect to $P$ by setting $\frac{\mathrm{d} Q}{\mathrm{~d} P}:=v$. Thereby (4.1) may be reformulated as

$$
\mathcal{E}_{0}^{g}(\xi)=\sup _{Q \ll P}\left\{E_{Q}[\xi]-\mathcal{E}_{0}^{*}(Q)\right\}, \quad \xi \in L^{1},
$$

where

$$
\mathcal{E}_{0}^{*}(Q):=\sup _{\xi \in L^{1}}\left\{E_{Q}[\xi]-\mathcal{E}_{0}^{g}(\xi)\right\} .
$$

Note that $\mathcal{A}(\xi, g, z)=\emptyset$ implies $\mathcal{E}_{0}^{g}(\xi)=+\infty$ and hence such terminal conditions are irrelevant for the supremum in (4.3). Let us denote by $\mathcal{Q}$ the set of all probability measures equivalent to $P$ with bounded Radon-Nikodym derivative. For each $Q \in \mathcal{Q}$, there exists a progressively measurable process $q$ taking values in $\mathbb{R}^{1 \times d}$ such that for all $t \in[0, T]$

$$
\left.\frac{\mathrm{d} Q}{\mathrm{~d} P}\right|_{\mathcal{F}_{t}}=\exp \left(\int_{0}^{t} q_{u} \mathrm{~d} W_{u}-\frac{1}{2} \int_{0}^{t}\left|q_{u}\right|^{2} \mathrm{~d} u\right) .
$$

By Girsanov's theorem, the process $W_{t}^{Q}:=W_{t}-\int_{0}^{t} q_{u} \mathrm{~d} u$ is a $Q$-Brownian motion. The following lemma is a valuable tool regarding the characterization of $\mathcal{E}_{0}^{*}$.

Lemma 4.2. The supremum in (4.3) can be restricted to random variables $\xi \in L^{1}$ for which the BSDE with parameters $(\xi, g)$ has a solution with value process starting in $\mathcal{E}_{0}^{g}(\xi)$. More precisely, for any $Q \in \mathcal{Q}$ holds

$$
\mathcal{E}_{0}^{*}(Q)=\sup _{\xi \in L^{1}}\left\{E_{Q}[\xi]-\mathcal{E}_{0}^{g}(\xi): B S D E(\xi, g) \text { has a solution }(Y, Z) \text { with } Y_{0}=\mathcal{E}_{0}^{g}(\xi)\right\} \text {. }
$$

Proof. It suffices to show that

$$
\mathcal{E}_{0}^{*}(Q) \leq \sup _{\xi \in L^{1}}\left\{E_{Q}[\xi]-\mathcal{E}_{0}^{g}(\xi): \operatorname{BSDE}(\xi, g) \text { has a solution }(Y, Z) \text { with } Y_{0}=\mathcal{E}_{0}^{g}(\xi)\right\}
$$

${ }^{7}$ Note that the case $\mathcal{E}_{0}^{g} \equiv+\infty$ on $L^{1}$ immediately yields $\mathcal{E}_{0}^{*} \equiv-\infty$ on $L^{\infty}$ and is thus neglected. 
since the reverse inequality is satisfied by definition of $\mathcal{E}_{0}^{*}(\cdot)$. Consider to this end a terminal condition $\xi \in L^{1}$ with associated minimal supersolution $(Y, Z) \in \mathcal{A}(\xi, g, z)$, that is $Y_{0}=\mathcal{E}_{0}^{g}(\xi)$. Put, for all $t \in[0, T]$,

$$
Y_{t}^{1}=\mathcal{E}_{0}^{g}(\xi)-\int_{0}^{t} g_{u}\left(Z_{u}, \Delta_{u}, \Gamma_{u}\right) \mathrm{d} u+\int_{0}^{t} Z_{u} \mathrm{~d} W_{u} .
$$

Relation (3.1) implies $Y_{T}^{1} \geq Y_{T} \geq \xi$ and thus $\mathcal{E}_{0}^{g}\left(Y_{T}^{1}\right) \geq \mathcal{E}_{0}^{g}(\xi)$ and $\left(Y_{T}^{1}\right)^{-} \in L^{1}$. Furthermore, observe that

$$
\left(Y_{T}^{1}\right)^{+}=\left(\mathcal{E}_{0}^{g}(\xi)-\int_{0}^{T} g_{u}\left(Z_{u}, \Delta_{u}, \Gamma_{u}\right) \mathrm{d} u+\int_{0}^{T} Z_{u} \mathrm{~d} W_{u}\right)^{+} \leq\left(\mathcal{E}_{0}^{g}(\xi)+\int_{0}^{T} Z_{u} \mathrm{~d} W_{u}\right)^{+}
$$

due to the positivity of the generator. But since the right-hand side is in $L^{1}$ by means of the martingale property of $\int Z \mathrm{~d} W$, we deduce that $\left(Y_{T}^{1}\right)^{+} \in L^{1}$, allowing us to conclude that $Y_{T}^{1} \in L^{1}$. On the other hand, $\left(Y^{1}, Z\right) \in$ $\mathcal{A}\left(Y_{T}^{1}, g, z\right)$ holds by definition of $Y^{1}$. Hence, we conclude $\mathcal{E}_{0}^{g}\left(Y_{T}^{1}\right) \leq Y_{0}^{1}=\mathcal{E}_{0}^{g}(\xi)$. Thus, $\mathcal{E}_{0}^{g}\left(Y_{T}^{1}\right)=\mathcal{E}_{0}^{g}(\xi)$, and $\left(Y^{1}, Z\right)$ is a solution of the BSDE with parameters $\left(Y_{T}^{1}, g\right)$. Observe further that $\mathcal{E}_{0}^{g}\left(Y_{T}^{1}\right)-\mathcal{E}_{0}^{g}(\xi)=0 \leq Y_{T}^{1}-\xi$ which, by taking expectation under $Q$, implies

$$
E_{Q}[\xi]-\mathcal{E}_{0}^{g}(\xi) \leq E_{Q}\left[Y_{T}^{1}\right]-\mathcal{E}_{0}^{g}\left(Y_{T}^{1}\right)
$$

Taking the supremum yields (4.4), the proof is done.

By means of the preceding lemma it holds

$$
\begin{aligned}
\mathcal{E}_{0}^{*}(Q) & =\sup _{\xi \in L^{1}}\left\{E_{Q}[\xi]-\mathcal{E}_{0}^{g}(\xi)\right\} \\
& =\sup _{\xi \in L^{1}}\left\{E_{Q}\left[\mathcal{E}_{0}^{g}(\xi)-\int_{0}^{T} g_{u}\left(Z_{u}, \Delta_{u}, \Gamma_{u}\right) \mathrm{d} u+\int_{0}^{T} Z_{u} \mathrm{~d} W_{u}\right]-\mathcal{E}_{0}^{g}(\xi)\right\} \\
& =\sup _{(\Delta, \Gamma) \in \Pi}\left\{E_{Q}\left[-\int_{0}^{T} g_{u}\left(Z_{u}, \Delta_{u}, \Gamma_{u}\right) \mathrm{d} u+\int_{0}^{T} Z_{u} \mathrm{~d} W_{u}\right]\right\}
\end{aligned}
$$

where

$$
\Pi:=\left\{(\Delta, \Gamma) \in \mathcal{L}^{2} \times \mathcal{L}^{2}: \begin{array}{l}
\exists \xi \in L^{1}: \operatorname{BSDE}(\xi, g) \text { has a solution }(Y, Z) \\
\text { with } Y_{0}=\mathcal{E}_{0}^{g}(\xi) \text { and } Z=z+\int \Delta \mathrm{d} u+\int \Gamma \mathrm{d} W
\end{array}\right\} .
$$

Whenever $Q \in \mathcal{Q}$, Girsanov's theorem applies and we may exploit the decomposition of $Z$ and use that $\int Z \mathrm{~d} W^{Q}$ and $\int \Gamma \mathrm{d} W^{Q}$ are $Q$-martingales in order to express the right-hand side of (4.5) without Brownian integrals. More precisely,

$$
\mathcal{E}_{0}^{*}(Q)=\sup _{(\Delta, \Gamma) \in \Pi}\left\{E_{Q}\left[\int_{0}^{T}\left(-g_{u}\left(Z_{u}, \Delta_{u}, \Gamma_{u}\right)+q_{u} \int_{0}^{u}\left(\Delta_{s}+q_{s} \Gamma_{s}\right) \mathrm{d} s\right) \mathrm{d} u\right]\right\}+z E_{Q}\left[\int_{0}^{T} q_{u} \mathrm{~d} u\right] .
$$

We continue with two lemmata that allow us to restrict the set of measures in the representation (4.2) to a sufficiently nice subset of $\mathcal{Q}$ on the one hand, and to change the set $\Pi$ appearing in (4.5) to the whole space $\mathcal{L}^{2} \times \mathcal{L}^{2}$ on the other hand.

Lemma 4.3. Assume there exists some $\xi \in L^{1}$ such that $\mathcal{A}(\xi, g, z) \neq \emptyset$. Then it is sufficient to consider measures with densities that are bounded away from zero, that is

$$
\mathcal{E}_{0}^{g}(\xi)=\sup _{v \in L_{b}^{\infty}}\left\{E[v \xi]-\mathcal{E}_{0}^{*}(v)\right\}
$$

where $L_{b}^{\infty}:=\left\{v \in L^{\infty}: v>0\right.$ and $\left.\left\|\frac{1}{v}\right\|_{\infty}<\infty\right\}$. 
Proof. The assumption of $\mathcal{A}(\xi, g, z)$ being non-empty for some $\xi \in L^{1}$ implies the existence of $(\Delta, \Gamma) \in \Pi$ and corresponding $Z$ such that $E_{P}\left[\int_{0}^{T} g_{u}\left(Z_{u}, \Delta_{u}, \Gamma_{u}\right) \mathrm{d} u\right]<\infty$ which together with (POS), (4.5) and the martingale property of all occurring $\int Z \mathrm{~d} W$ under $P$ immediately yields that $\mathcal{E}_{0}^{*}(P)<\infty$. For any $Q \ll P$ with $\frac{\mathrm{d} Q}{\mathrm{~d} P}=$ $v \in L_{+}^{\infty}$ and $\lambda \in(0,1)$ we define a measure $Q^{\lambda}$ by its Radon-Nikodym derivative $v_{\lambda}:=(1-\lambda) v+\lambda$ where naturally $\frac{\mathrm{d} P}{\mathrm{~d} P}=1$. Observe that $\lambda>0$ implies $v_{\lambda} \in L_{b}^{\infty}$. Next, we show that $\lim _{\lambda \downarrow 0} \mathcal{E}_{0}^{*}\left(v_{\lambda}\right)=\mathcal{E}_{0}^{*}(v)$. Indeed, convexity of $\mathcal{E}_{0}^{*}(\cdot)$ together with $\mathcal{E}_{0}^{*}\left(\frac{\mathrm{d} P}{\mathrm{~d} P}\right)=\mathcal{E}_{0}^{*}(1)<\infty$ yields $\lim \inf _{\lambda \downarrow 0} \mathcal{E}_{0}^{*}\left(v_{\lambda}\right) \leq \mathcal{E}_{0}^{*}(v)$, whereas the reverse inequality is satisfied by means of the lower semicontinuity. On the other hand, dominated convergence gives $\lim _{\lambda \downarrow 0} E\left[v_{\lambda} \xi\right]=E[v \xi]$, since $\left|v_{\lambda} \xi\right| \leq|v \xi|+|\xi|$ which is integrable. Consequently, the expression $\left\{E[v \xi]-\mathcal{E}_{0}^{*}(v)\right\}$ is the limit of a sequence $\left(E\left[v_{\lambda_{n}} \xi\right]-\mathcal{E}_{0}^{*}\left(v_{\lambda_{n}}\right)\right)_{n}$ where $\left(v_{\lambda_{n}}\right) \subset L_{b}^{\infty}$ and $\lambda_{n} \downarrow$. Since $\mathcal{E}_{0}^{g}(\xi)$ can be expressed as the supremum of $\left\{E[v \xi]-\mathcal{E}_{0}^{*}(v)\right\}$ over all $v$, it suffices to consider the supremum over $v \in L_{b}^{\infty}$, the proof is done.

Lemma 4.4. For each $Q \in \mathcal{Q}$ such that $\frac{\mathrm{d} Q}{\mathrm{~d} P} \in L_{b}^{\infty}$ it holds

$$
\mathcal{E}_{0}^{*}(Q)=\sup _{(\Delta, \Gamma) \in \mathcal{L}^{2} \times \mathcal{L}^{2}}\left\{E_{Q}\left[-\int_{0}^{T} g_{u}\left(Z_{u}, \Delta_{u}, \Gamma_{u}\right) \mathrm{d} u+\int_{0}^{T} Z_{u} \mathrm{~d} W_{u}\right]\right\} .
$$

Proof. Since $\Pi$ defined in (4.6) is a subset of $\mathcal{L}^{2} \times \mathcal{L}^{2}$, " $\leq$ " certainly holds in (4.9). As to the reverse inequality, observe first that, since we consider a supremum in (4.9) and $Z \in \mathcal{L}^{2}$ whenever $(\Delta, \Gamma) \in \mathcal{L}^{2} \times \mathcal{L}^{2}$, those $(\Delta, \Gamma)$ such that $E_{Q}\left[\int_{0}^{T} g_{u}\left(Z_{u}, \Delta_{u}, \Gamma_{u}\right) \mathrm{d} u\right]=+\infty$ can be neglected in the following. In particular, since $v=\frac{\mathrm{d} Q}{\mathrm{~d} P} \in L_{b}^{\infty}$, we can restrict our focus to those elements satisfying $E\left[\int_{0}^{T} g_{u}\left(Z_{u}, \Delta_{u}, \Gamma_{u}\right) \mathrm{d} u\right] \leq\left\|\frac{1}{v}\right\|_{L^{\infty}} E_{Q}\left[\int_{0}^{T} g_{u}\left(Z_{u}, \Delta_{u}, \Gamma_{u}\right) \mathrm{d} u\right]<+\infty$. Thus, given such a pair $(\Delta, \Gamma)$, the terminal condition $\xi:=-\int_{0}^{T} g_{u}\left(Z_{u}, \Delta_{u}, \Gamma_{u}\right) \mathrm{d} u+\int_{0}^{T} Z_{u} \mathrm{~d} W_{u}$ fulfills $\xi^{-} \in L^{1}$ due to the martingale property of $\int Z \mathrm{~d} W$. Furthermore, the pair $\left(-\int_{0}^{*} g_{u}\left(Z_{u}, \Delta_{u}, \Gamma_{u}\right) \mathrm{d} u+\int_{0}^{*} Z_{u} \mathrm{~d} W_{u}, Z\right)$ is an element of $\mathcal{A}(\xi, g, z)$ by construction and hence Theorem 3.7 yields the existence of $(\bar{Y}, \bar{Z}) \in \mathcal{A}(\xi, g, z)$ satisfying $\bar{Y}_{0}=\mathcal{E}_{0}^{g}(\xi) \leq 0$. Now, using the same techniques as in the proof of Lemma 4.2 , we define $Y^{1}$ by $Y_{t}^{1}:=\mathcal{E}_{0}^{g}(\xi)-\int_{0}^{t} g_{u}\left(\bar{Z}_{u}, \bar{\Delta}_{u}, \bar{\Gamma}_{u}\right) \mathrm{d} u+\int_{0}^{t} \bar{Z}_{u} \mathrm{~d} W_{u}$, for all $t \in[0, T]$, where $(\bar{\Delta}, \bar{\Gamma})$ is the decomposition of $\bar{Z}$, and obtain that $Y_{T}^{1} \geq \xi$ as well as $\mathcal{E}_{0}^{g}\left(Y_{T}^{1}\right)=\mathcal{E}_{0}^{g}(\xi)$. Consequently,

$$
-\int_{0}^{T} g_{u}\left(\bar{Z}_{u}, \bar{\Delta}_{u}, \bar{\Gamma}_{u}\right) \mathrm{d} u+\int_{0}^{T} \bar{Z}_{u} \mathrm{~d} W_{u}=Y_{T}^{1}-\mathcal{E}_{0}^{g}(\xi) \geq Y_{T}^{1} \geq \xi=-\int_{0}^{T} g_{u}\left(Z_{u}, \Delta_{u}, \Gamma_{u}\right) \mathrm{d} u+\int_{0}^{T} Z_{u} \mathrm{~d} W_{u},
$$

which, by taking expectation under $Q$ in (4.10) and using $(\bar{\Delta}, \bar{\Gamma}) \in \Pi$, implies

$$
\mathcal{E}_{0}^{*}(Q) \geq E_{Q}\left[-\int_{0}^{T} g_{u}\left(Z_{u}, \Delta_{u}, \Gamma_{u}\right) \mathrm{d} u+\int_{0}^{T} Z_{u} \mathrm{~d} W_{u}\right] .
$$

Since $(\Delta, \Gamma)$ was arbitrary, we have finally shown that $\mathcal{E}_{0}^{*}(Q)$ is greater or equal to the supremum over $(\Delta, \Gamma) \in$ $\mathcal{L}^{2} \times \mathcal{L}^{2}$ of the right-hand side of (4.11), which finishes the proof.

The ensuing proposition provides, for a given measure $Q \in \mathcal{Q}$ with $\frac{\mathrm{d} Q}{\mathrm{~d} P} \in L_{b}^{\infty}$, the existence of a pair of processes attaining the supremum in (4.5).

Proposition 4.5. For each $Q \in \mathcal{Q}$ with $\frac{\mathrm{d} Q}{\mathrm{~d} P} \in L_{b}^{\infty}$ there exist $\left(\Delta^{Q}, \Gamma^{Q}\right) \in \Pi$ and a corresponding control $Z^{Q}$ of the form $Z^{Q}=z+\int \Delta^{Q} \mathrm{~d} u+\int \Gamma^{Q} \mathrm{~d} W$ such that

$$
\mathcal{E}_{0}^{*}(Q)=E_{Q}\left[-\int_{0}^{T} g_{u}\left(Z_{u}^{Q}, \Delta_{u}^{Q}, \Gamma_{u}^{Q}\right) \mathrm{d} u+\int_{0}^{T} Z_{u}^{Q} \mathrm{~d} W_{u}\right] .
$$

Furthermore, if the convexity of $g$ is strict, then the triple $\left(Z^{Q}, \Delta^{Q}, \Gamma^{Q}\right)$ is unique. 
Proof.

Step 1: The integral $\int q \mathrm{~d} W$ is an element of $B M O$. We begin by proving that, for $Q \in \mathcal{Q}$ the density $\frac{\mathrm{d} Q}{\mathrm{~d} P}=\exp \left(\int_{0}^{T} q_{u} \mathrm{~d} W_{u}-\frac{1}{2} \int_{0}^{T}\left|q_{u}\right|^{2} \mathrm{~d} u\right)$ of which belongs to $L_{b}^{\infty}$, the process $\left(\int_{0}^{t} q_{u} \mathrm{~d} W_{u}\right)_{t \in[0, T]}$ is an element of $B M O$. Indeed, since the process $v_{t}:=E\left[\frac{\mathrm{d} Q}{\mathrm{~d} P} \mid \mathcal{F}_{t}\right]$ is uniformly bounded away from zero, it satisfies the Muckenhaupt $\left(A_{1}\right)$ condition (see [16], Def. 2.2), and therefore $\int q \mathrm{~d} W \in B M O$ by means of ([16], Thm. 2.4).

Step 2: $\mathcal{L}^{2}$-boundedness of a minimizing sequence and the candidate $\left(\Delta^{Q}, \Gamma^{Q}\right)$. Since the generator $g$ satisfies (DGC), it holds for all $(\Delta, \Gamma, Z)$ that

$$
\|\Delta\|_{\mathcal{L}^{2}(Q)}^{2}+\|\Gamma\|_{\mathcal{L}^{2}(Q)}^{2} \leq \frac{1}{c_{2}}\left(E_{Q}\left[\int_{0}^{T} g_{u}\left(Z_{u}, \Delta_{u}, \Gamma_{u}\right) \mathrm{d} u\right]-c_{1} T\right) .
$$

If we put $F(Z, \Delta, \Gamma):=E_{Q}\left[\int_{0}^{T}\left(g_{u}\left(Z_{u}, \Delta_{u}, \Gamma_{u}\right) \mathrm{d} u-q_{u} \int_{0}^{u}\left(\Delta_{s}+q_{s} \Gamma_{s}\right) \mathrm{d} s\right) \mathrm{d} u\right]$, then (4.7) in combination with Lemma 4.4 implies that the conjugate can be expressed by $\mathcal{E}_{0}^{*}(Q)=-\inf _{(\Delta, \Gamma) \in \mathcal{L}^{2} \times \mathcal{L}^{2}} F(Z, \Delta, \Gamma)+z E_{Q}\left[\int_{0}^{T} q_{u} \mathrm{~d} u\right]$. We claim that, for $\left(Z^{n}, \Delta^{n}, \Gamma^{n}\right)$ a minimizing sequence of $F$, both $\left(\Delta^{n}\right)$ and $\left(\Gamma^{n}\right)$ are bounded in $\mathcal{L}^{2}(Q)$. Since in our case the $\mathcal{L}^{2}$-norms with respect to $P$ and $Q$ are equivalent, we suppress the dependence on the measure in the notation to follow. Assume now contrary to our assertion that $\left\|\Delta^{n}\right\|_{\mathcal{L}^{2}}^{2} \rightarrow \infty$ and $\left\|\Gamma^{n}\right\|_{\mathcal{L}^{2}}^{2} \rightarrow \infty$ as $n$ tends to infinity. This in turn would imply either

$$
E_{Q}\left[\int_{0}^{T} q_{u} \int_{0}^{u} \Delta_{s}^{n} \mathrm{~d} s \mathrm{~d} u\right] \rightarrow \infty \quad \text { and } \quad \limsup _{n} \frac{\left\|\Delta^{n}\right\|_{\mathcal{L}^{2}}^{2}}{E_{Q}\left[\int_{0}^{T} q_{u} \int_{0}^{u} \Delta_{s}^{n} \mathrm{~d} s \mathrm{~d} u\right]}=K
$$

or

$$
E_{Q}\left[\int_{0}^{T} q_{u} \int_{0}^{u} q_{s} \Gamma_{s}^{n} \mathrm{~d} s \mathrm{~d} u\right] \rightarrow \infty \quad \text { and } \quad \limsup _{n} \frac{\left\|\Gamma^{n}\right\|_{\mathcal{L}^{2}}^{2}}{E_{Q}\left[\int_{0}^{T} q_{u} \int_{0}^{u} q_{s} \Gamma_{s}^{n} \mathrm{~d} s \mathrm{~d} u\right]}=L
$$

or both, for $K, L \in \mathbb{R}$. Indeed, (4.13) would otherwise lead to $\lim _{n} F\left(Z^{n}, \Delta^{n}, \Gamma^{n}\right)=\infty$ and thereby contradict $\left(\left(Z^{n}, \Delta^{n}, \Gamma^{n}\right)\right)$ being a minimizing sequence of $F$. On the other hand however, an application of Hölder's inequality yields

$$
\begin{aligned}
\left|E_{Q}\left[\int_{0}^{T} q_{u} \int_{0}^{u} \Delta_{s}^{n} \mathrm{~d} s \mathrm{~d} u\right]\right| & \leq\left(E_{Q}\left[\int_{0}^{T}\left|q_{u}\right|^{2} \mathrm{~d} u\right] E_{Q}\left[\int_{0}^{T}\left(\int_{0}^{u} \Delta_{s}^{n} \mathrm{~d} s\right)^{2} \mathrm{~d} u\right]\right)^{\frac{1}{2}} \\
& \leq\|q\|_{\mathcal{L}^{2}}\left(E_{Q}\left[\int_{0}^{T} \int_{0}^{u}\left|\Delta_{s}^{n}\right|^{2} \mathrm{~d} s \mathrm{~d} u\right]\right)^{\frac{1}{2}} \leq T^{\frac{1}{2}}\|q\|_{\mathcal{L}^{2}}\left\|\Delta^{n}\right\|_{\mathcal{L}^{2}} .
\end{aligned}
$$

Taking the square on both sides above we obtain

$$
\left(E_{Q}\left[\int_{0}^{T} q_{u} \int_{0}^{u} \Delta_{s}^{n} \mathrm{~d} s \mathrm{~d} u\right]\right)^{2} \leq T\|q\|_{\mathcal{L}^{2}}^{2}\left\|\Delta^{n}\right\|_{\mathcal{L}^{2}}^{2}
$$


which in turn implies $\left\|\Delta^{n}\right\|_{\mathcal{L}^{2}}^{2}\left(E_{Q}\left[\int_{0}^{T} q_{u} \int_{0}^{u} \Delta_{s}^{n} \mathrm{~d} s \mathrm{~d} u\right]\right)^{-1} \rightarrow \infty$, a contradiction to (4.14). As to $\left(\Gamma^{n}\right)$, we argue similarly and, for $\left(Q_{u}\right)_{u \in[0, T]}$ defined by $Q_{u}:=\int_{u}^{T} q_{s} \mathrm{~d} s$ estimate

$$
\begin{aligned}
\left|E_{Q}\left[\int_{0}^{T} q_{u} \int_{0}^{u} q_{s} \Gamma_{s}^{n} \mathrm{~d} s \mathrm{~d} u\right]\right| & =\mid E_{Q}\left[\int_{0}^{T} q_{u} \Gamma_{u}^{n} Q_{u} \mathrm{~d} u\right] \leq E_{Q}\left[\int_{0}^{T}\left|q_{u} \| \Gamma_{u}^{n}\right|\left|Q_{u}\right| \mathrm{d} u\right] \\
& \leq\left(E_{Q}\left[\int_{0}^{T}\left|q_{u}\right|^{2}\left|Q_{u}\right|^{2} \mathrm{~d} u\right]\right)^{\frac{1}{2}}\left\|\Gamma^{n}\right\|_{\mathcal{L}^{2}}=\left(E_{Q}\left[\left.\int_{0}^{T}\left|q_{u}\right|^{2}\left|\int_{u}^{T} q_{s} \mathrm{~d} s\right|\right|^{2} \mathrm{~d} u\right]\right)^{\frac{1}{2}}\left\|\Gamma^{n}\right\|_{\mathcal{L}^{2}} \\
& \leq\left(E_{Q}\left[\left(\int_{0}^{T}\left|q_{u}\right|^{2} \mathrm{~d} u\right)^{2}\right]\right)^{\frac{1}{2}}\left\|\Gamma^{n}\right\|_{\mathcal{L}^{2}}=\|q\|_{\mathcal{L}^{4}}^{2}\left\|\Gamma^{n}\right\|_{\mathcal{L}^{2}}
\end{aligned}
$$

where we used Fubini's theorem in the first equality above. Since $\int q \mathrm{~d} W \in B M O$, the $\mathcal{L}^{4}$-norm of $q$ is finite ${ }^{8}$ and the contradiction to (4.15) is derived analogously to the argumentation following (4.16). Consequently, there exists a sequence $\left(\left(\tilde{\Delta}^{n}, \tilde{\Gamma}^{n}\right)\right)$ in the asymptotic convex hull of $\left(\left(\Delta^{n}, \Gamma^{n}\right)\right)$ and $\left(\Delta^{Q}, \Gamma^{Q}\right) \in \mathcal{L}^{2} \times \mathcal{L}^{2}$ such that $\left(\left(\tilde{\Delta}^{n}, \tilde{\Gamma}^{n}\right)\right)$ converges in $\mathcal{L}^{2} \times \mathcal{L}^{2}$ to $\left(\Delta^{Q}, \Gamma^{Q}\right)$. On the side of $\left(Z^{n}\right)$ we pass to the corresponding sequence $\left(\tilde{Z}^{n}\right)$ and recall from the proof of Lemma 3.4 that it is bounded in $\mathcal{L}^{2}$. Hence, there is a sequence in the asymptotic convex hull of $\left(\tilde{Z}^{n}\right)$, denoted likewise, that converges in $\mathcal{L}^{2}$ to some $Z^{Q}=z+\int \Delta^{Q} \mathrm{~d} u+\int \Gamma^{Q} \mathrm{~d} W$. Of course, we pass the corresponding sequence on the side of $\left(\left(\tilde{\Delta}^{n}, \tilde{\Gamma}^{n}\right)\right)$ without violating the convergence to $\left(\Delta^{Q}, \Gamma^{Q}\right)$.

Step 3: Lower Semicontinuity and convexity of $F$. In a next step we show that the earlier defined function $F(Z, \Delta, \Gamma)=E_{Q}\left[\int_{0}^{T} g_{u}\left(Z_{u}, \Delta_{u}, \Gamma_{u}\right) \mathrm{d} u-\int_{0}^{T} Z_{u} \mathrm{~d} W_{u}\right]$ is lower semicontinuous and convex on $\mathcal{L}^{2} \times \mathcal{L}^{2} \times \mathcal{L}^{2}$ where $Z=z+\int \Delta \mathrm{d} u+\int \Gamma \mathrm{d} W$. Indeed, the part $E_{Q}\left[\int_{0}^{T} g_{u}\left(Z_{u}, \Delta_{u}, \Gamma_{u}\right) \mathrm{d} u\right]$ is lower semicontinuous by (POS), (LSC) and Fatou's lemma. As to the second part, first observe that $\mathcal{L}^{2}$-convergence of $\left(\tilde{Z}^{n}\right)$ towards $Z^{Q}$ implies

$$
\left|E_{Q}\left[\int_{0}^{T}\left(\tilde{Z}_{u}^{n}-Z_{u}^{Q}\right) \mathrm{d} W_{u}\right]\right| \underset{n \rightarrow \infty}{\longrightarrow} 0 .
$$

Furthermore, (CON) yields that $F$ is convex in $(Z, \Delta, \Gamma)$.

Step 4: Minimality of $\left(Z^{Q}, \Delta^{Q}, \Gamma^{Q}\right)$. We claim that $F\left(Z^{Q}, \Delta^{Q}, \Gamma^{Q}\right)=\inf _{(\Delta, \Gamma) \in \mathcal{L}^{2} \times \mathcal{L}^{2}} F(Z, \Delta, \Gamma)$ which would then in turn finally imply (4.12). To this end, it suffices to prove that $F\left(Z^{Q}, \Delta^{Q}, \Gamma^{Q}\right) \leq$ $\inf _{(\Delta, \Gamma) \in \mathcal{L}^{2} \times \mathcal{L}^{2}} F(Z, \Delta, \Gamma)$, since the reverse inequality is naturally satisfied. Observe now that

$$
\begin{aligned}
\inf _{(\Delta, \Gamma) \in \mathcal{L}^{2} \times \mathcal{L}^{2}} F(Z, \Delta, \Gamma) & =\lim _{n} F\left(Z^{n}, \Delta^{n}, \Gamma^{n}\right)=\lim _{n} \sum_{k=n}^{M(n)} \lambda_{k}^{(n)} F\left(Z^{k}, \Delta^{k}, \Gamma^{k}\right) \\
& \geq \lim _{n} F\left(\sum_{k=n}^{M(n)} \lambda_{k}^{(n)} Z^{k}, \sum_{k=n}^{M(n)} \lambda_{k}^{(n)} \Delta^{k}, \sum_{k=n}^{M(n)} \lambda_{k}^{(n)} \Gamma^{k}\right) \\
& =\lim _{n} F\left(\tilde{Z}^{n}, \tilde{\Delta}^{n}, \tilde{\Gamma}^{n}\right) \geq F\left(Z^{Q}, \Delta^{Q}, \Gamma^{Q}\right)
\end{aligned}
$$

where we denoted by $\lambda_{k}^{(n)}, n \leq k \leq M(n), \sum_{k} \lambda_{k}^{(n)}=1$ the convex weights of the sequence $\left(\left(\tilde{Z}^{n}, \tilde{\Delta}^{n}, \tilde{\Gamma}^{n}\right)\right)$ and made use of the convexity and lower semicontinuity of $F$.

\footnotetext{
${ }^{8}$ Recall that $B M O$ can be embedded into any $\mathcal{H}^{p}$-space, compare ([16], Sect. 2.1, p. 26).
} 
Step 5: Uniqueness of $\left(Z^{Q}, \Delta^{Q}, \Gamma^{Q}\right)$. As to the uniqueness, assume that there are $\left(\Delta^{1}, \Gamma^{1}\right)$ and $\left(\Delta^{2}, \Gamma^{2}\right)$ with corresponding $Z^{1}$ and $Z^{2}$, respectively, both attaining the supremum such that $P \otimes \mathrm{d} t\left[\left(\Delta^{1}, \Gamma^{1}\right) \neq\left(\Delta^{2}, \Gamma^{2}\right)\right]>0$. Setting $(\bar{Z}, \bar{\Delta}, \bar{\Gamma}):=\frac{1}{2}\left[\left(Z^{1}, \Delta^{1}, \Gamma^{1}\right)+\left(Z^{2}, \Delta^{2}, \Gamma^{2}\right)\right]$ together with $Q \sim P$ and the strict convexity of $F$ inherited by $g$ yields that $F(\bar{Z}, \bar{\Delta}, \bar{\Gamma})<F\left(Z^{1}, \Delta^{1}, \Gamma^{1}\right)$, a contradiction to the optimality of $\left(Z^{1}, \Delta^{1}, \Gamma^{1}\right)$.

Remark 4.6. Since for a given $Q \in \mathcal{Q}$ with $\frac{\mathrm{d} Q}{\mathrm{~d} P} \in L_{b}^{\infty}$ and a strictly convex generator the maximizer $\left(Z^{Q}, \Delta^{Q}, \Gamma^{Q}\right)$ is unique by the preceding proposition, it has to be (conditionally) optimal at all times $t \in[0, T]$. Indeed, assume to the contrary the existence of $(\Delta, \Gamma)$ such that $z+\int_{0}^{t} \Delta_{u} \mathrm{~d} u+\int_{0}^{t} \Gamma_{u} \mathrm{~d} W_{u}=Z_{t}=Z_{t}^{Q}$ and $E_{Q}\left[\int_{t}^{T}-g_{u}\left(Z_{u}, \Delta_{u}, \Gamma_{u}\right) \mathrm{d} u+\int_{t}^{T} Z_{u} \mathrm{~d} W_{u} \mid \mathcal{F}_{t}\right]>E_{Q}\left[\int_{t}^{T}-g_{u}\left(Z_{u}^{Q}, \Delta_{u}^{Q}, \Gamma_{u}^{Q}\right) \mathrm{d} u+\int_{t}^{T} Z_{u}^{Q} \mathrm{~d} W_{u} \mid \mathcal{F}_{t}\right]$ holds true for some $t \in[0, T]$. Then, however, the concatenated processes $(\bar{Z}, \bar{\Delta}, \bar{\Gamma}):=\left(Z^{Q}, \Delta^{Q}, \Gamma^{Q}\right) 1_{[0, t]}+(Z, \Delta, \Gamma) 1_{] t, T]}$ satisfy $E_{Q}\left[\int_{0}^{T}-g_{u}\left(\bar{Z}_{u}, \bar{\Delta}_{u}, \bar{\Gamma}_{u}\right) \mathrm{d} u+\int_{0}^{T} \bar{Z}_{u} \mathrm{~d} W_{u}\right]>E_{Q}\left[\int_{0}^{T}-g_{u}\left(Z_{u}^{Q}, \Delta_{u}^{Q}, \Gamma_{u}^{Q}\right) \mathrm{d} u+\int_{0}^{T} Z_{u}^{Q} \mathrm{~d} W_{u}\right]$, which is a contradiction to the opimality of $\left(Z^{Q}, \Delta^{Q}, \Gamma^{Q}\right)$ at time zero.

Notice that, for $d=1$ and the case of a quadratic generator which is in addition independent of $z$, that is $g_{u}(\delta, \gamma)=|\delta|^{2}+|\gamma|^{2}$, the processes $\left(\Delta^{Q}, \Gamma^{Q}\right)$ attaining $\mathcal{E}_{0}^{*}(Q)$ can be explicitly computed and $\left(\Delta_{t}^{Q}, \Gamma_{t}^{Q}\right)$ depends on the whole path of $q$ up to time $t$, as illustrated in the following proposition. It thus constitutes a useful tool for the characterization of the dual optimizers and its proof is closely related to the Euler-Lagrange equation arising in classical calculus of variation.

Proposition 4.7. Assume that $d=1$ and that $g$ is defined by $g_{u}(\delta, \gamma)=|\delta|^{2}+|\gamma|^{2}$. For $Q \in \mathcal{Q}$ with $\frac{\mathrm{d} Q}{\mathrm{~d} P} \in L_{b}^{\infty}$, let $\left(\Delta^{Q}, \Gamma^{Q}\right)$ be the optimizer attaining $\mathcal{E}_{0}^{*}(Q)$. Then there exist $c_{1}, c_{2} \in \mathbb{R}$ such that

$$
\begin{aligned}
\Delta_{t}^{Q} & =-\frac{1}{2} \int_{0}^{t} q_{s} \mathrm{~d} s+c_{1} \\
\Gamma_{t}^{Q} & =-\frac{1}{2} q_{t}\left(\int_{0}^{t} q_{s} \mathrm{~d} s+c_{2}\right),
\end{aligned}
$$

for all $t \in[0, T]$.

Proof. For the purpose of this proof we assume without loss of generality that $z=0$, since the initial value does not affect the optimization with respect to $(\Delta, \Gamma)$. Hence, the generator only depending on $\delta$ and $\gamma$ in combination with (4.7) gives $\mathcal{E}_{0}^{*}(Q)=-\inf _{(\Delta, \Gamma)}\left\{F_{1}(\Delta)+F_{2}(\Gamma)\right\}$ where $F_{1}(\Delta):=E_{Q}\left[\int_{0}^{T}\left(\left|\Delta_{u}\right|^{2}-q_{u} \int_{0}^{u} \Delta_{s} \mathrm{~d} s\right) \mathrm{d} u\right]$ and $F_{2}(\Gamma):=E_{Q}\left[\int_{0}^{T}\left(\left|\Gamma_{u}\right|^{2}-q_{u} \int_{0}^{u} q_{s} \Gamma_{s} \mathrm{~d} s\right) \mathrm{d} u\right]$. We will proceed along an $\omega$-wise criterion of optimality, since any pair $\left(\Delta^{Q}, \Gamma^{Q}\right)$ that is optimal for almost all $\omega \in \Omega$ then naturally also optimizes the expectation under $Q$. The uniqueness obtained in Proposition 4.5 then assures that the path-wise optimizer is the only one. We define

$$
J_{1}(\Delta)=\int_{0}^{T}\left(\left|\Delta_{u}\right|^{2}-q_{u} \int_{0}^{u} \Delta_{s} \mathrm{~d} s\right) \mathrm{d} u \quad \text { and } \quad J_{2}(\Gamma)=\int_{0}^{T}\left(\left|\Gamma_{u}\right|^{2}-q_{u} \int_{0}^{u} q_{s} \Gamma_{s} \mathrm{~d} s\right) \mathrm{d} u
$$

and observe that it is sufficient to elaborate how to obtain conditions for a minimizer of $J_{1}$, as the functional $J_{2}$ is of a similar structure. Introducing $X(u):=\int_{0}^{u} \Delta_{s} \mathrm{~d} s$ we obtain $X^{\prime}(u):=\frac{\mathrm{d}}{\mathrm{d} u} X(u)=\Delta_{u}$ and

$$
J_{1}(\Delta)=\tilde{J}_{1}(X)=\int_{0}^{T} L\left(u, X(u), X^{\prime}(u)\right) \mathrm{d} u
$$

where $L(u, a, b)=|b|^{2}-q_{u} a$. If $X$ is a local minimum of $\tilde{J}_{1}$, then $\tilde{J}_{1}(X) \leq \tilde{J}_{1}(X+\varepsilon \eta)$ for sufficiently small $\varepsilon>0$ and all differentiable $\eta \in C([0, T], \mathbb{R})$ the derivatives of which are square integrable and which satisfy $\eta(0)=\eta(T)=0$. In particular, with $\phi(\varepsilon):=\tilde{J}_{1}(X+\varepsilon \eta)$, it has to hold that $\frac{\mathrm{d}}{\mathrm{d} \varepsilon} \phi(\varepsilon)_{\left.\right|_{\varepsilon=0}}=0$. Using the specific 
form of $L$ we get

$$
\begin{aligned}
\frac{\mathrm{d}}{\mathrm{d} \varepsilon} \phi(\varepsilon)_{\left.\right|_{\varepsilon=0}} & =\lim _{h \rightarrow 0} \frac{1}{h} \int_{0}^{T}\left[L\left(u, X(u)+h \eta(u), X^{\prime}(u)+h \eta^{\prime}(u)\right)-L\left(u, X(u), X^{\prime}(u)\right)\right] \mathrm{d} u \\
& =\lim _{h \rightarrow 0} \int_{0}^{T}\left[-q_{u} \eta(u)+2 X^{\prime}(u) \eta^{\prime}(u)+h\left(\eta^{\prime}(u)\right)^{2}\right] \mathrm{d} u .
\end{aligned}
$$

Having assumed $\eta^{\prime}$ to be square integrable allows us to exchange limit and integration, yielding

$$
0=\frac{\mathrm{d}}{\mathrm{d} \varepsilon} \phi(\varepsilon)_{\left.\right|_{\varepsilon=0}}=\int_{0}^{T}\left[-q_{u} \eta(u)+2 \Delta_{u} \eta^{\prime}(u)\right] \mathrm{d} u .
$$

Using integration by parts we obtain

$$
-\int_{0}^{T} q_{u} \eta(u) \mathrm{d} u=\left.\left(\int_{0}^{u}-q_{s} \mathrm{~d} s\right) \eta(u)\right|_{0} ^{T}-\int_{0}^{T}\left(\int_{0}^{u}-q_{s} \mathrm{~d} s\right) \eta^{\prime}(u) \mathrm{d} u .
$$

The first term on the right-hand side above vanishes and so, by plugging this back into (4.19) we end up with

$$
\int_{0}^{T}\left(2 \Delta_{u}+\int_{0}^{u} q_{s} \mathrm{~d} s\right) \eta^{\prime}(u) \mathrm{d} u=0 .
$$

Let us next introduce the constant $c:=\frac{1}{T} \int_{0}^{T}\left(2 \Delta_{u}+\int_{0}^{u} q_{s} \mathrm{~d} s\right) \mathrm{d} u$, of course depending on $\omega$, and observe that, using $\int_{0}^{T} \eta^{\prime}(u) \mathrm{d} u=0$, Equation (4.20) may be rewritten as

$$
\int_{0}^{T}\left(2 \Delta_{u}+\int_{0}^{u} q_{s} \mathrm{~d} s-c\right) \eta^{\prime}(u) \mathrm{d} u=0 .
$$

Moreover, the function

$$
\bar{\eta}(t):=\int_{0}^{t}\left(2 \Delta_{u}+\int_{0}^{u} q_{s} \mathrm{~d} s-c\right) \mathrm{d} u
$$

satisfies $\bar{\eta}(0)=\bar{\eta}(T)=0$ by construction as well as $\bar{\eta}^{\prime}(u)=2 \Delta_{u}+\int_{0}^{u} q_{s} \mathrm{~d} s-c$, which is square integrable for almost all $\omega \in \Omega$, since $\Delta$ and $q$ are square integrable ${ }^{9}$. Hence, (4.21) applied to our particular function $\bar{\eta}$ yields

$$
\int_{0}^{T}\left(2 \Delta_{u}+\int_{0}^{u} q_{s} \mathrm{~d} s-c\right)^{2} \mathrm{~d} u=0
$$

and we deduce that

$$
2 \Delta_{u}+\int_{0}^{u} q_{s} \mathrm{~d} s=\frac{1}{T} \int_{0}^{T}\left(2 \Delta_{r}+\int_{0}^{r} q_{s} \mathrm{~d} s\right) d r \quad \text { for almost all } u \in[0, T] .
$$

Specifically, (4.22) shows that, for almost all $\omega \in \Omega$, there exists a set $\mathcal{I}(\omega) \subseteq[0, T]$ with Lebesgue measure $T$ such that, for all $u \in \mathcal{I}(\omega)$,

$$
2 \Delta_{u}+\int_{0}^{u} q_{s} \mathrm{~d} s=M
$$

where $M$ of course depends on $\omega \in \Omega$ and is thus a random variable. This in turn implies, for $\mathrm{d} t$-almost all $u \in[0, T]$, the existence of $\Omega_{u} \subseteq \Omega$ with $P\left(\Omega_{u}\right)=1$ such that (4.23) holds for all $\omega \in \Omega_{u}$. In particular, on $\Omega_{u}$ the above $M$ equals an $\mathcal{F}_{u}$-measurable random variable. We choose a sequence $\left(u_{n}\right) \subset[0, T]$ with $\lim _{n} u_{n}=0$

\footnotetext{
${ }^{9}$ More precisely, it holds $P\left(\int_{0}^{T}\left|q_{u}\right|^{2} \mathrm{~d} u<\infty\right)=P\left(\int_{0}^{T}\left|\Delta_{u}\right|^{2} \mathrm{~d} u<\infty\right)=1$.
} 
and hence obtain that on $\bar{\Omega}:=\bigcap_{n} \Omega_{u_{n}}$, where $P(\bar{\Omega})=Q(\bar{\Omega})=1, M$ equals an $\bigcap_{n} \mathcal{F}_{u_{n}}$-measurable random variable and is thus $\mathcal{F}_{0}$-measurable, that means it is a constant on $\bar{\Omega}$, by the right-continuity of our filtration. Since modifying our optimizer on a $Q$-nullset does not alter the value of the functional to be optimized, we have shown (4.17) by putting $c_{1}:=\frac{M}{2}$.

As to the case of our optimal $\Gamma^{Q}$, assume first that $q_{u} \neq 0$ for all $u \in[0, T]$ and observe that, with $Y(u):=\int_{0}^{u} q_{s} \Gamma_{s} \mathrm{~d} s$, we obtain $J_{2}(\Gamma)=\tilde{J}_{2}(Y)=\int_{0}^{T} K\left(u, Y(u), Y^{\prime}(u)\right) \mathrm{d} u$ where we set $K(u, a, b)=$ $\left(1 / q_{u}\right)^{2} b^{2}-q_{u} a$. Thus, an argumentation identical to that above would yield (4.18) in that case. Furthermore, it holds that $\lim _{q_{u} \rightarrow 0} \Gamma_{u}^{Q}=0$, a value that is consistent with the "pointwise" minimization consideration that $\left.\arg \min _{\Gamma_{u}}\left\{\left|\Gamma_{u}\right|^{2}-q_{u} \int_{[0, u)} q_{s} \Gamma_{s} \mathrm{~d} s\right\}\right|_{q_{u}=0}=0$ and hence justifies expression (4.18).

For $\xi \in L^{1}$ such that $\mathcal{A}(\xi, g, z) \neq \emptyset$, the following theorem states that, given the existence of an equivalent probability measure $\hat{Q} \in \mathcal{Q}$ such that the sup in (4.2) is attained, the BSDE with generator $g$ and terminal condition $\xi$ admits a solution under constraints. Conditions guaranteeing that the rather technical assumptions of the ensuing theorem are satisfied are subject to further research.

Theorem 4.8. Assume that, for $\xi \in L^{1}$ with $\mathcal{A}(\xi, g, z) \neq \emptyset$, there exists $a \hat{Q} \in \mathcal{Q}$ with $\frac{\mathrm{d} Q}{\mathrm{~d} P} \in L_{b}^{\infty}$ such that $\mathcal{E}_{0}^{g}(\xi)=E_{\hat{Q}}[\xi]-\mathcal{E}_{0}^{*}(\hat{Q})$. Then there exists a solution $(Y, Z) \in \mathcal{A}(\xi, g, z)$ of the BSDE with parameters $(\xi, g)$.

Proof. Starting with (4.2) in combination with Proposition 4.5, it holds

$$
\mathcal{E}_{0}^{g}(\xi)=E_{\hat{Q}}[\xi]-\mathcal{E}_{0}^{*}(\hat{Q})=E_{\hat{Q}}\left[\xi+\int_{0}^{T} g_{u}\left(Z_{u}^{\hat{Q}}, \Delta_{u}^{\hat{Q}}, \Gamma_{u}^{\hat{Q}}\right) \mathrm{d} u-\int_{0}^{T} Z_{u}^{\hat{Q}} \mathrm{~d} W_{u}\right] .
$$

We recall that $\mathcal{A}(\xi, g, z) \neq \emptyset$ and $\xi \in L^{1}$. Hence, by Theorem 3.7 there exists $(\Delta, \Gamma, Z)$ such that

$$
E_{\hat{Q}}\left[\xi+\int_{0}^{T} g_{u}\left(Z_{u}^{\hat{Q}}, \Delta_{u}^{\hat{Q}}, \Gamma_{u}^{\hat{Q}}\right) \mathrm{d} u-\int_{0}^{T} Z_{u}^{\hat{Q}} \mathrm{~d} W_{u}\right]-\int_{0}^{T} g_{u}\left(Z_{u}, \Delta_{u}, \Gamma_{u}\right) \mathrm{d} u+\int_{0}^{T} Z_{u} \mathrm{~d} W_{u} \geq \xi
$$

holds true. Taking expectation under $\hat{Q}$ on both sides of the inequality above yields

$$
E_{\hat{Q}}\left[-\int_{0}^{T} g_{u}\left(Z_{u}^{\hat{Q}}, \Delta_{u}^{\hat{Q}}, \Gamma_{u}^{\hat{Q}}\right) \mathrm{d} u+\int_{0}^{T} Z_{u}^{\hat{Q}} \mathrm{~d} W_{u}\right] \leq E_{\hat{Q}}\left[-\int_{0}^{T} g_{u}\left(Z_{u}, \Delta_{u}, \Gamma_{u}\right) \mathrm{d} u+\int_{0}^{T} Z_{u} \mathrm{~d} W_{u}\right] .
$$

However, the expression on the left-hand side is maximal for $\left(Z^{\hat{Q}}, \Delta^{\hat{Q}}, \Gamma^{\hat{Q}}\right)$ by means of Proposition 4.5 and thus equality has to hold. Hence, it follows that $\mathcal{E}_{0}^{*}(\hat{Q})=E_{\hat{Q}}\left[-\int_{0}^{T} g_{u}\left(Z_{u}, \Delta_{u}, \Gamma_{u}\right) \mathrm{d} u+\int_{0}^{T} Z_{u} \mathrm{~d} W_{u}\right]$. By plugging this back into (4.24) we obtain

$$
\mathcal{E}_{0}^{g}(\xi)=E_{\hat{Q}}[\xi]+E_{\hat{Q}}\left[\int_{0}^{T} g_{u}\left(Z_{u}, \Delta_{u}, \Gamma_{u}\right) \mathrm{d} u-\int_{0}^{T} Z_{u} \mathrm{~d} W_{u}\right]
$$

which is equivalent to

$$
E_{\hat{Q}}\left[\mathcal{E}_{0}^{g}(\xi)-\int_{0}^{T} g_{u}\left(Z_{u}, \Delta_{u}, \Gamma_{u}\right) \mathrm{d} u+\int_{0}^{T} Z_{u} \mathrm{~d} W_{u}-\xi\right]=0 .
$$

Since the expression within the expectation is $P$ - and thereby also $\hat{Q}$-almost surely positive, we finally conclude that

$$
\mathcal{E}_{0}^{g}(\xi)-\int_{0}^{T} g_{u}\left(Z_{u}, \Delta_{u}, \Gamma_{u}\right) \mathrm{d} u+\int_{0}^{T} Z_{u} \mathrm{~d} W_{u}=\xi
$$

and thus $\left(\mathcal{E}_{0}^{g}(\xi)-\int_{0}^{\cdot} g(Z, \Delta, \Gamma) \mathrm{d} u+\int_{0}^{*} Z \mathrm{~d} W, Z\right)$ constitutes a solution of the BSDE with parameters $(\xi, g)$. 


\section{REFERENCES}

[1] P. Briand and Y. Hu, BSDE with quadratic growth and unbounded terminal value. Probab. Theory Relat. Fields 136 (2006) 604-618.

[2] M. Broadie, J. Cvitanic and H.M. Soner, Optimal Replication of Contingent Claims under Portfolio Constraints. Rev. Financ. Stud. 11 (1998) 59-79.

[3] P. Cheridito, H.M. Soner and N. Touzi, The multi-dimensional super-replication problem under gamma constraints. Ann. Inst. Henri Poincaré, Analyse Non Linéaire 22 (2005) 633-666.

[4] P. Cheridito, H.M. Soner, N. Touzi and N. Victoir, Second Order Backward Stochastic Differential Equations and Fully NonLinear Parabolic PDEs. Commun. Pure Appl. Math. 60 (2007) 1081-1110.

[5] J. Cvitanic and I. Karatzas, Hedging contingent claims with constrained portfolios. Ann. Appl. Probab. 3 (1993) $652-681$.

[6] F. Delbaen, Y. Hu and X. Bao, Backward SDEs with Superquadratic Growth. Probab. Theory Relat. Fields 150 (2011) $145-192$.

[7] F. Delbaen and W. Schachermayer, A General Version of the Fundamental Theorem of Asset Pricing. Math. Annalen 300 (1994) 463-520.

[8] F. Delbaen and W. Schachermayer, A Compactness Principle for Bounded Sequences of Martingales with Applications. Proc. of the Seminar of Stochastic Analysis, Random Fields and Applications. Progress in Probability. Birkhäuser (1996) 133-173.

[9] S. Drapeau, G. Heyne and M. Kupper, Minimal Supersolutions of Convex BSDEs. Ann. Probab. 41 (2013) 3973-4001.

[10] S. Drapeau, M. Kupper, E.R. Gianin and L. Tangpi, Dual Representation of Minimal Supersolutions of Convex BSDEs. Ann. Inst. Henri Poincaré 52 (2016) 868-887.

[11] N. El Karoui, S. Peng and M. C. Quenez, Backward Stochastic Differential Equations in Finance. Math. Finance 7 (1997) $1-71$.

[12] G. Heyne, Essays on Minimal Supersolutions of BSDEs and on Cross Hedging in Incomplete Markets. Ph.D. thesis, HumboldtUniversität zu Berlin (2012).

[13] G. Heyne, M. Kupper and C. Mainberger, Minimal Supersolutions of BSDEs with Lower Semicontinuous Generators. Ann. Inst. Henri Poincaré, Probabilités Statistiques 50 (2014) 524-538.

[14] E. Jouini and H. Kallal, Arbitrage in securities markets with short-sales constraints. Math. Finance 5 (1995) $197-232$.

[15] I. Karatzas and S.E. Shreve, Brownian Motion and Stochastic Calculus. Graduate Texts in Mathematics. Springer (2004).

[16] N. Kazamaki, Continuous Exponential Martingales and BMO. Vol. 1579 of Lect. Notes Math.. Springer-Verlag, Berlin (1994). ISBN 3-540-58042-5.

[17] M. Kobylanski, Backward Stochastic Differential Equations and Partial Differential Equations with Quadratic Growth. Ann. Probab. 28 (2000) 558-602.

[18] E. Pardoux and S. Peng, Adapted Solution of a Backward Stochastic Differential Equation. System Control Lett. 14 (1990) 55-61.

[19] S. Peng, Monotonic Limit Theorem of BSDE and Nonlinear Decomposition Theorem of Doob-Meyer's Type. Probab. Theory Relat. Fields 113 (1999) 473-499.

[20] P.E. Protter, Stochastic Integration and Differential Equations, 2nd edition. Springer (2005).

[21] H.M. Soner and N. Touzi, Superreplication under Gamma constraints. SIAM J. Control Optim. 39 (2000) 73-96.

[22] H.M. Soner, N. Touzi and J. Zhang, Dual Formulation of Second Order Target Problems. Ann. Appl. Probab. 23 (2013) 308-347. 